ARTICLE

\title{
DNA methylation study of Huntington's disease and motor progression in patients and in animal models
}

Ake T. Lu (1) 1,10, Pritika Narayan (10 2,10, Matthew J. Grant ${ }^{2}$, Peter Langfelder ${ }^{3,4}$, Nan Wang ${ }^{3,4}$, Seung Kwak ${ }^{5}$, Hilary Wilkinson (1) ${ }^{5}$, Richard Z. Chen ${ }^{5}$, Jian Chen (1) ${ }^{5}$, C. Simon Bawden ${ }^{6}$, Skye R. Rudiger ${ }^{6}$, Marc Ciosi ${ }^{7}$, Afroditi Chatzi ${ }^{7}$, Alastair Maxwell7, Timothy A. Hore (i) ${ }^{8}$, Jeff Aaronson ${ }^{5}$, Jim Rosinski ${ }^{5}$, Alicia Preiss ${ }^{5}$, Thomas F. Vogt ${ }^{5}$, Giovanni Coppola (1) ${ }^{3,4}$, Darren Monckton (D) ${ }^{7}$, Russell G. Snell ${ }^{2,11}$, X. William Yang (i) ${ }^{3,4,11}$ \& Steve Horvath (10) 1,9,11凶

Although Huntington's disease (HD) is a well studied Mendelian genetic disorder, less is known about its associated epigenetic changes. Here, we characterize DNA methylation levels in six different tissues from 3 species: a mouse huntingtin ( $\mathrm{Htt}$ ) gene knock-in model, a transgenic HTT sheep model, and humans. Our epigenome-wide association study (EWAS) of human blood reveals that HD mutation status is significantly $\left(p<10^{-7}\right)$ associated with 33 CpG sites, including the HTT gene $\left(p=6.5 \times 10^{-26}\right)$. These $H t t / H T T$ associations were replicated in the Q175 $\mathrm{Htt}$ knock-in mouse model $\left(p=6.0 \times 10^{-8}\right)$ and in the transgenic sheep model $\left(p=2.4 \times 10^{-88}\right)$. We define a measure of HD motor score progression among manifest HD cases based on multiple clinical assessments. EWAS of motor progression in manifest HD cases exhibits significant $\left(p<10^{-7}\right)$ associations with methylation levels at three loci: near PEX14 $\left(p=9.3 \times 10^{-9}\right)$, GRIK4 $\left(p=3.0 \times 10^{-8}\right)$, and COX4I2 $\left(p=6.5 \times 10^{-8}\right)$. We conclude that HD is accompanied by profound changes of DNA methylation levels in three mammalian species.

\footnotetext{
${ }^{1}$ Department of Human Genetics, David Geffen School of Medicine, University of California, Los Angeles, Los Angeles, CA 90095, USA. ${ }^{2}$ Applied Translational Genetics Group, School of Biological Sciences, Centre for Brain Research, The University of Auckland, Auckland 1010, New Zealand. ${ }^{3}$ Center for Neurobehavioral Genetics, Jane and Terry Semel Institute for Neuroscience and Human Behavior, University of California, Los Angeles (UCLA), Los Angeles, CA 90095, USA. ${ }^{4}$ Department of Psychiatry and Biobehavioral Sciences, David Geffen School of Medicine at UCLA, Los Angeles, CA 90095, USA. ${ }^{5}$ CHDI Management/CHDI Foundation, Princeton, NJ 08540, USA. ${ }^{6}$ Livestock and Farming Systems, South Australian Research and Development Institute, Roseworthy, SA 5371, Australia. ${ }^{7}$ Institute of Molecular, Cell and Systems Biology, College of Medical, Veterinary and Life Sciences, University of Glasgow, Glasgow G12 8QQ, UK. ${ }^{8}$ Department of Anatomy, University of Otago, Dunedin 9016, New Zealand. ${ }^{9}$ Department of Biostatistics, School of Public Health, University of California, Los Angeles, Los Angeles, CA 90095, USA. ${ }^{10}$ These authors contributed equally: Ake T. Lu, Pritika Narayan. ${ }^{11}$ These authors jointly supervised this work: Russell G. Snell, X.William Yang, Steve Horvath. ${ }^{凶}$ email: shorvath@mednet.ucla.edu
} 
untington's disease (HD) is a dominantly inherited neurodegenerative disorder clinically characterized by a progressive movement disorder, cognitive dysfunction, and psychiatric impairment. HD gene-expansion carriers (HDGECs) have CAG-repeat lengths of 36 or greater on one of the alleles of the huntingtin (HTT) gene. HD is one of several polyglutamine disorders (including spinal cerebellar ataxias types $1,2,3,6$, and 7 , spinal and bulbar muscular atrophy, and dentatorubral-pallidoluysian atrophy) that are caused by the expansion of unstable CAG trinucleotide repeats ${ }^{1}$.

Since these disorders exhibit distinct patterns of neuronal loss and clinical manifestation, the differential pathogenesis of polyglutamine disorders may be due to differences in polyglutamine protein context or the host protein function. This is despite nearly ubiquitous expression of these proteins, at least in the brain, and, in the case of HTT, expression throughout the body and during development. The age of onset of motor (AMO) symptoms is strongly and inversely correlated with the number of CAG trinucleotide repeats in $H T T^{2-4}$. A recent study showed that HTT CAG-repeat length but not polyglutamine length determines the timing of HD motor onset ${ }^{5}$. HD patients are usually clinically diagnosed in their 40s, but the AMO ranges from younger than 10 years for individuals with highrepeat lengths to, in rare cases, over 80 years. Age plays an important role in $\mathrm{HD}$; for example, the product of CAG-repeat length and chronological age (CAP score) relates to clinical progression in $\mathrm{HD}$ according to longitudinal studies of HDGEC cohorts ${ }^{3}$.

By exploiting DNA methylation (DNAm)-based biomarker of tissue age (referred to as the epigenetic clock), we have shown that $\mathrm{HD}$ is associated with epigenetic age acceleration (AgeAccel) and greatly disrupted changes in DNAm levels in brain tissues ${ }^{6}$. Recent studies have looked at methylation levels of selected genes in HDGECs $^{7}$ and analyzed 13 human cortical samples ${ }^{8}$. DNA (de)methylation in an HTT gene context has also been investigated in transgenic animal models ${ }^{9}$. However, it is not yet known whether HD is associated with significant DNAm changes in other human tissues and in transgenic animal models.

Here we present, to our knowledge, the largest DNAm study of $\mathrm{HD}$ to date. We generate large methylation datasets from three species and from eight sources of DNA. We evaluate composite epigenetic biomarkers and individual CpGs in terms of their relationship with (a) HDGEC status and (b) HD progression in individuals with manifest motor symptoms. Our epigenetic clock study demonstrates that manifest HD is associated with increased epigenetic age in human blood DNA. Epigenome-wide association studies (EWAS) of HD mutation status reveal that HTT is the most significant locus in multiple tissues from three species. Blood DNAm levels of select genomic loci are associated with motor score progression among manifest HD patients.

\section{Results}

Our methylation study datasets derive from three species: (I) a total of 2164 human samples across blood, lymphoblast, and fibroblast tissues collected from five datasets, (II) a total of 112 mouse tissues (cerebellum, striatum, blood, cortex, and liver) measured on two platforms (reduced representation bisulfite sequencing (RRBS) and a custom methylation array), and (III) 168 blood samples from sheep. All methylation datasets are described in Table 1 (for human) and Supplementary Table 1 (for mouse and sheep). Human blood samples were a subset of a larger collection from Enroll-HD ${ }^{10}$ and Registry-HD $\mathrm{HD}^{11}$ datasets; the longitudinal clinical measures from the subset of cases were used to perform the HD progression analysis.

Human data. We generated five human DNAm datasets using the Illumina Infinium array $(N=2164$ samples; Table 1): (1)
$N=910$ blood samples from Enroll-HD study (data 1); (2) $N=$ 714 longitudinal blood samples from 357 individuals from EnrollHD (data 2); (3) $N=376$ blood samples from Registry-HD; (4) $N=100$ lymphoblastoid cell samples from Registry-HD; and (5) $N=64$ fibroblast samples from the CHDI. The Enroll-HD study datasets (blood data 1 and 2) used in this study were a subset of a larger collection of 33,288 observations from 15,203 individuals ("Methods"; Supplementary Tables 2, 3). Apart from studying the effect of HTT mutation status on DNAm levels, we also tested the hypothesis that methylation levels in blood-derived DNA are predictive of motor progression in manifest $\mathrm{HD}$ cases. We analyzed large-scale longitudinal motor score assessments from (1) Enroll-HD data 1 after the initial blood draw (on average 3.1 years of follow-up; Supplementary Table 2), (2) the Enroll-HD data 2, which only involved HDGECs (on average 7.9 years apart from the first blood draw; Supplementary Table 3), and (3) the last visit in the Registry-HD cohort (Table 1). Our EWAS of HD progression involved 917 individuals with manifest HD for whom both DNAm profiles and longitudinal clinical assessments were available ("Methods").

HD motor progression in manifest HD cases. A linear mixed analysis was performed to define a measure of HD motor progression in manifest patients from Enroll-HD. For data 1, we used a large-scale Enroll-HD database comprising 14,850 longitudinal observations across 5204 manifest patients ("Methods"). Total motor scores were measured by the Unified Huntington's Disease Rating Scale (UHDRS) ${ }^{12}$, a standard clinical assessment to quantify the severity of disease (Supplementary Note 1). A higher UHDRS motor score indicates a more severe disease progression. Figure 1a illustrates the heterogeneity of slopes of longitudinal motor scores versus visit, indicating the utility of modeling visit as a random effect in a linear mixed-effects model. A linear mixedeffects model was used to relate the longitudinal motor scores (dependent variable) to visit (random effect), age, sex, CAG length, age at motor onset, and educational attainment ("Methods"). Our measure of HD progression was defined by the random slope estimate after adjusting for the other covariates. The adjusted random slope estimate can be viewed as a measure of atypical HD progression not predicted by HTT mutation, age, and potential confounders. This measure is analogous to the one used in an earlier genome-wide association study (GWAS) of HD progression $^{13}$.

After $>2$ years of follow-up, motor scores were substantially increased (on average 7.6 points; Supplementary Fig. 1). As expected, CAG-repeat length and AMO were significantly associated with an increase in motor score in a linear mixed model analysis (Table 2). The model revealed that motor scores were increased by $2.7 \pm 0.09\left(p=5.3 \times 10^{-191}\right)$ per one unit increase in CAG-repeat length, increased by $2.2 \pm 0.05\left(p<5.0 \times 10^{-300}\right)$ per year (of age), increased by $3.5 \pm 0.08\left(p<5.0 \times 10^{-300}\right)$ per year of follow-up, and decreased by $1.54 \pm 0.05\left(p=1.24 \times 10^{-201}\right)$ per year of AMO. Females were associated with higher motor scores $(p=$ $1.16 \times 10^{-12}$ ) and years of education were associated with lower motor scores $\left(p=3.31 \times 10^{-22}\right)$.

A linear mixed analysis was analogously performed in EnrollHD data 2 (1867 observations across $N=278$ patients, with a longer follow-up period ("Methods" and Supplementary Table 4). The motor score in this group at the last visit showed a substantially larger increase (on average 27.43 compared to 7.6), due to the longer follow-up period (on average 7.8 years compared to 2 years; Supplementary Fig. 1). Both linear mixedeffects models reveal that our measures of HD motor progression (random slope) are significantly associated with age and CAGrepeat length in both Enroll-HD data 1 and 2 (Table 2 and 
Table 1 A total of 2164 methylation samples across blood, lymphoblastoid, and fibroblast tissues were used for human crosssectional studies.

\begin{tabular}{|c|c|c|c|c|c|}
\hline & \multicolumn{5}{|l|}{ Parameter } \\
\hline & Buffy coat & Buffy coat & Whole blood & Lymphoblastoid & Fibroblast \\
\hline \multicolumn{6}{|l|}{ No. of individuals } \\
\hline Control & 294 & 0 & 78 & 21 & 27 \\
\hline \multicolumn{6}{|l|}{ Age } \\
\hline HDGEC & $49[18,76]$ & $45[18,76]$ & $53[19,84]$ & $52[19,82]$ & $43[31,58]$ \\
\hline Control & $51[18,76]$ & - & $49[20,84]$ & $52[20,82]$ & $41[31,55]$ \\
\hline \multicolumn{6}{|l|}{ CAG length } \\
\hline HDGEC & $43[36,51]$ & $44[37,59]$ & $45[39,67]$ & $45[39,66]$ & $44[41,50]$ \\
\hline Controls & $20[16,35]$ & - & $21[14,33]$ & $21[17,31]$ & - \\
\hline Normalization method & Noob 57 & Noob $^{57}$ & $N_{o o b} 57$ & $N b^{57}$ & $N_{o o b} 57$ \\
\hline Data analysis & $\begin{array}{l}\text { Clock, EWAS, } \\
\text { regression }\end{array}$ & Clock, EWAS, regression & $\begin{array}{l}\text { Clock, EWAS, } \\
\text { regression }\end{array}$ & Clock, regression & Clock, regression \\
\hline $\begin{array}{l}\text { Columns 1-5 correspond to blood } \\
\text { collected (separated by roughly } 7 \\
\text { (EWAS), and multivariate regressi } \\
\text { last two columns correspond to no } \\
\text { to a single disease-Huntington's } \\
\text { Continuous parameters are preser } \\
\text { HDGEC refers to HD gene-expans } \\
\text { aHD status and age estimates rep }\end{array}$ & $\begin{array}{l}\text { on analysis. Both Enroll-HD } \\
\text { n-blood methylation data fr } \\
\text { disease. } \\
\text { ted in the format of mean } \\
\text { ion carriers (combining pr }\end{array}$ & 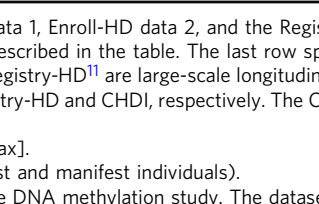 & $\begin{array}{l}\text { y-HD, respectively. } \\
\text { cifies the use of the } \\
\text { observational coho } \\
\text { DI Foundation is a p }\end{array}$ & $\begin{array}{l}\text { vidual in the Enroll-HD } \\
\text { enetic clock analysis, ep } \\
\text { g the onset and progres } \\
\text { d, not-for-profit biomedi }\end{array}$ & $\begin{array}{l}\text { o blood samples were } \\
\text { wide association study } \\
\text { intington's disease. The } \\
\text { ch organization devoted }\end{array}$ \\
\hline
\end{tabular}

Supplementary Table 4, respectively). CAG-repeat length exhibits particularly significant associations with $\mathrm{HD}$ progression $(p=$ $1.1 \times 10^{-13}$ in data 1 and $p=1.2 \times 10^{-7}$ in data 2 ).

In the Registry-HD data, we used a different definition for motor progression because it differed from Enroll-HD in terms of the assessment of follow-up information. Short-term follow-up information (on average $1.18 \pm 0.6$ years) was available for 224 out of 287 manifest patients from the Registry-HD study. Similar to a previous study of motor progression in the Registry-HD data $^{13}$, we defined progression as raw residual resulting from a linear model that regressed the motor score at the last visit (dependent variable) on age and other determinants of motor progression ("Methods"). The Registry-HD data showed comparable patterns for the associations of the fixed effects with motor scores in terms of directions of regression coefficients and effect sizes (Table 2 and Supplementary Table 5).

HD manifest patients exhibiting accelerated blood epigenetic aging effects. We applied five human epigenetic biomarkers of aging (epigenetic clocks) that lend themselves to analyzing blood methylation data: (1) Horvath's pan-tissue epigenetic age (referred to as DNAm age $\left.{ }^{14}\right)$; (2) Hannum's blood-based DNAm age ${ }^{15}$; (3) skin and blood clock (DNAmAgeSkinClock) ${ }^{16}$; (4) DNAmPhenoAge $^{17}$; and (5) the mortality risk estimator DNAmGrimAge ${ }^{18}$.

Using the longitudinal Enroll-HD data 2, we found that all DNAm age estimators track the passage of study time (Fig. 2a-d). In the cross-sectional data, the correlation between DNAm age estimators and chronological ages were $>0.84$, led by DNAmAgeSkinClock (Fig. 2c) with correlation estimates ranging between 0.95 and 0.97 across all the three datasets (Supplementary Figs. 2e, f, $3 \mathrm{c}$, and $4 \mathrm{c}$ ).
To formally measure possible epigenetic AgeAccel effects due to HD disease status, we fit a regression model of DNAm age on chronological age and defined AgeAccel as the resulting raw residual. Thus, positive AgeAccel means the methylation state of the sample appears to be older than would be expected based on chronological age. We found that manifest HD (but not pre-manifest HD) had significantly higher epigenetic AgeAccel than controls $\left(0.098 \leq p \leq 2.0 \times 10^{-4}\right.$, Fig. 3$)$, led by the pan-tissue clock (AgeAccel, $p=2.0 \times 10^{-4}$, Fig. 3a) and AgeAccelGrim $\left(p=2.4 \times 10^{-4} ;\right.$ Fig. $\left.3 f\right)$. The difference remained significant $\left(p=1.1 \times 10^{-3}\right.$; Fig. $\left.3 \mathrm{~b}\right)$ when studying the measure of intrinsic epigenetic AgeAccel (IEAA), which is independent of imputed blood cell counts ${ }^{19}$. Despite the low number of controls $(N=78)$, we managed to corroborate the finding for AgeAccelGrim in the Registry-HD data $(p=0.046$; Supplementary Fig. 5L).

A multivariate model analysis demonstrates that the observed epigenetic AgeAccel effects are not due to confounders (Supplementary Table 6). In particular, we find that manifest $\mathrm{HD}$ is associated with a significant increase in DNAm age $(p=$ $2.9 \times 10^{-3}$ ) in the Enroll-HD data even after adjusting for the 10 principal components. However, this association could not be replicated in the Registry-HD data (Supplementary Table 7).

The pan-tissue clock also revealed a strong correlation between lymphoblastoid cells blood ( $r=0.74, p=2.1 \times 10^{-18}$; Supplementary Fig. 6A), but the DNAm Age estimate was significantly lower than that of corresponding blood samples (mean difference $=3.3$ years, $p=0.0017$; Supplementary Fig. $6 \mathrm{~B}$ ), suggesting that the Epstein-Barr virus transformation used in generating lymphoblastoid cell lines decreases DNAm age.

Next, we tested whether epigenetic AgeAccel is associated with motor progression in manifest HD. The considered measures 
a

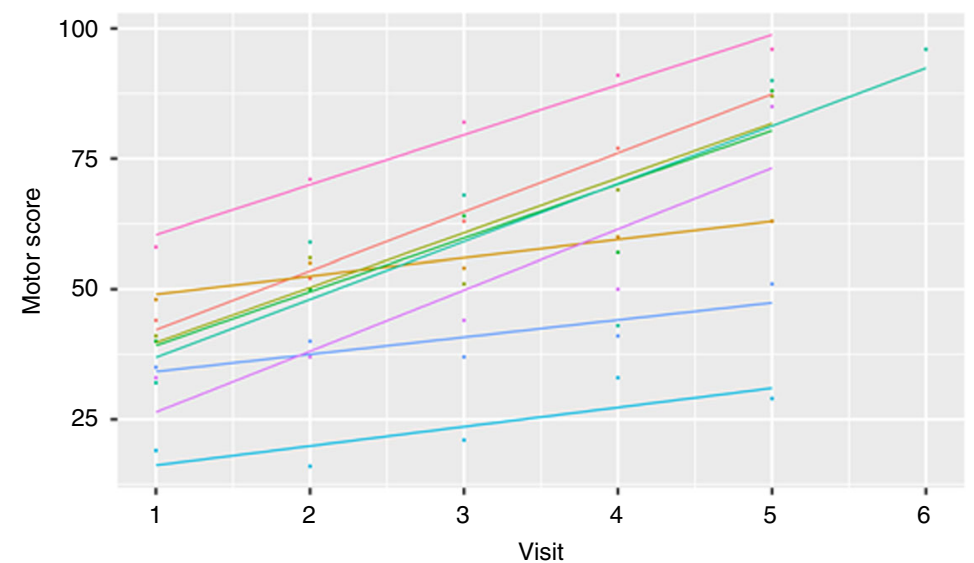

b

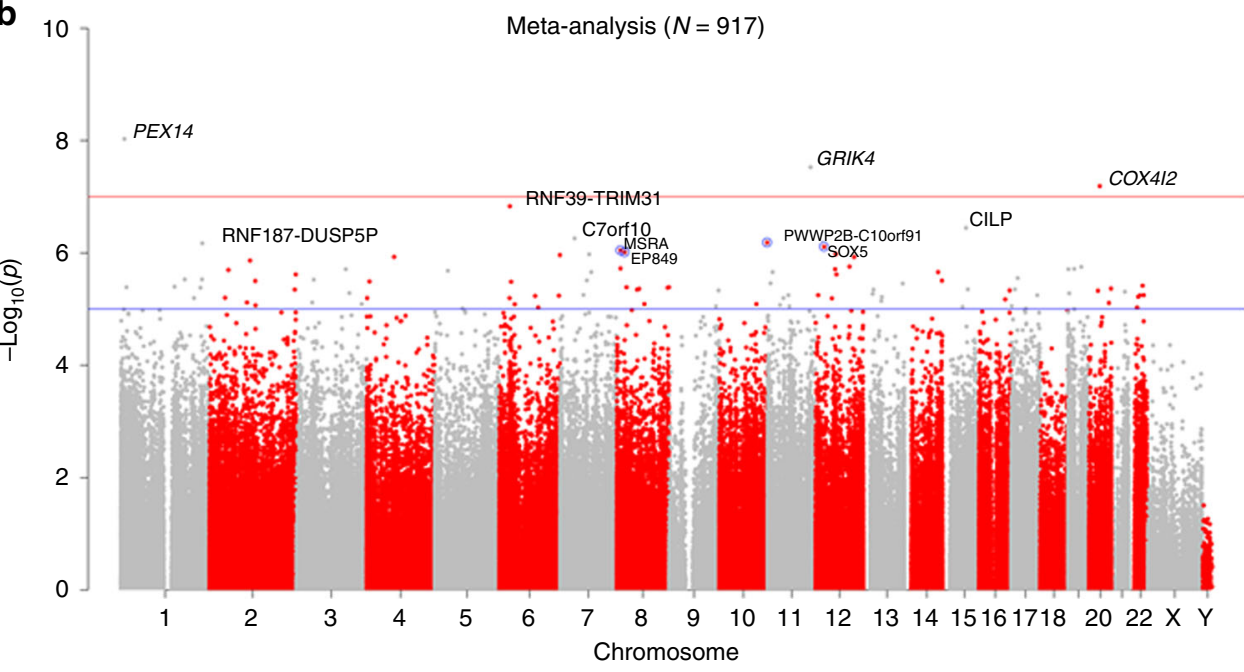

Fig. 1 EWAS of motor progression in manifest HD cases. a The spaghetti plot illustrates how the motor score depends on the visit in Enroll-HD. To arrive at a measure of motor score progression (i.e., the rate of change in the motor score), we estimated the slope using a random-effects model with a random slope and intercept term. $\mathbf{b}$ The Manhattan plot visualizes the EWAS results of motor progression in manifest HD cases: log (base 10)-transformed metaanalysis $p$ value ( $y$-axis) versus the chromosomal location of each $\mathrm{CpG}$ ( $x$-axis). The EWAS results were calculated with the biweight midcorrelation tests ${ }^{50}$. Fixed-effects meta-analysis (weighted by inverse variance) was performed to combine the EWAS results across blood data $(N=917)$ from EnrollHD data 1, Enroll-HD data 2, and Registry data. All $p$ values are two-sided and not adjusted for multiple comparisons. The blue and red horizontal lines correspond to suggestive significance $\left(\alpha=1.0 \times 10^{-5}\right)$ and genome-wide significant levels $\left(\alpha=1.0 \times 10^{-7}\right)$, respectively. Gene names are provided for CpGs (blue circles) with $p<1.0 \times 10^{-6}$ with detailed summary statistics reported in Supplementary Data 3.

\section{Table 2 Linear mixed model regression analysis for HD motor progression.}

\section{Parameter Outcome: motor score}

Parameter

\begin{tabular}{ll} 
& Coef. \\
\hline Female & 3.375
\end{tabular}

$\mathrm{Ag}$

CAG length

Age at motor onset

Education

Visit
0.474

$2.743-0.089$

$\begin{array}{rr}2.743 & 0.089 \\ -1.542 & 0.049\end{array}$

$-1.877 \quad 0.193$

$3.516 \quad 0.078$

\begin{tabular}{lc} 
SE & $\boldsymbol{T}$ statisti \\
\hline 0.474 & 7.127 \\
0.045 & 48.878 \\
0.089 & 30.757 \\
0.049 & -31.687 \\
0.193 & -9.742 \\
0.078 & 45.019
\end{tabular}

$\boldsymbol{P}$ value
$1.16 \times 10^{-12}$
$<5.0 \times 10^{-300}$
$5.27 \times 10^{-191}$
$1.24 \times 10^{-201}$
$3.09 \times 10^{-22}$
$<5.0 \times 10^{-300}$

Outcome: random slope

The table presents the coefficient estimates from two linear regression models: (1) a linear mixed model analysis of 14,850 longitudinal motor scores (dependent variable) across 5204 manifest HD cases from Enroll-HD data 1 and (2) a linear regression model analysis of the resulting random slope estimates (dependent variable) of the same 5204 cases. The linear mixed-effects model included two random-effects (a random intercept term and a random slope term with respect to visit) and several fixed-effect terms (sex, age at baseline, CAG-repeat length, age at motor onset, educational attainment). The empirical Bayes estimate of the random slope was used as a measure of HD motor progression for each of the $N=5204$ manifest HD cases. We adjusted the random slope estimate for all fixed effects in our downstream EWAS analysis. The columns report the covariate name, regression coefficient, standard error, Student's $T$ statistic, and unadjusted two-sided Wald test $p$ value. 
a

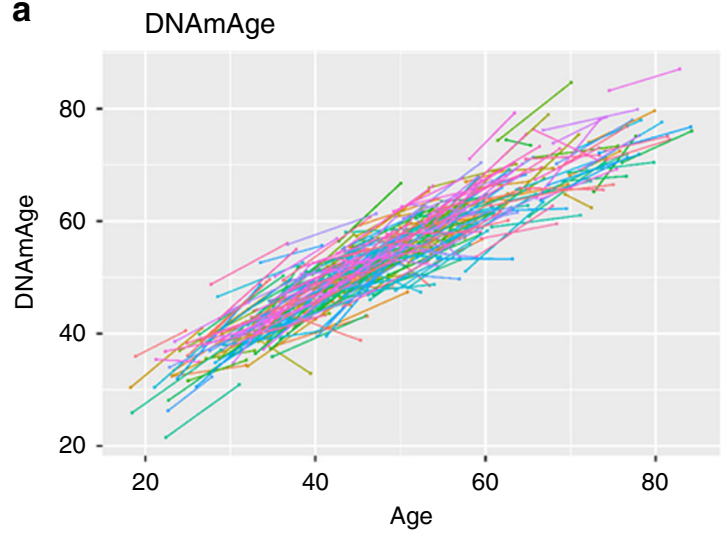

C

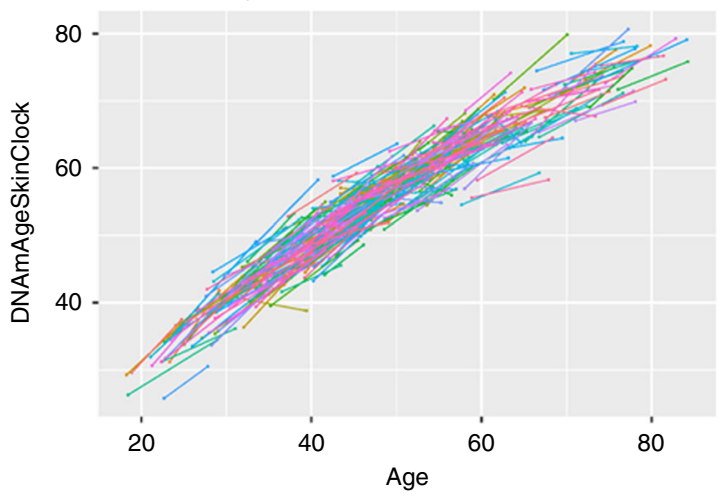

b

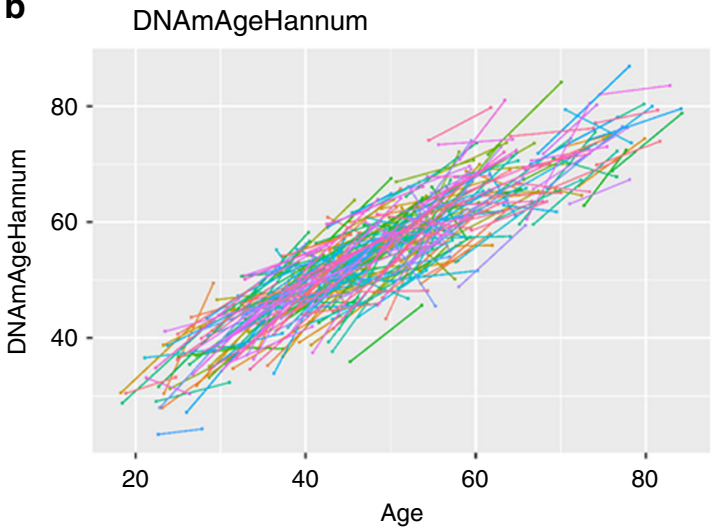

d

DNAmPhenoAge

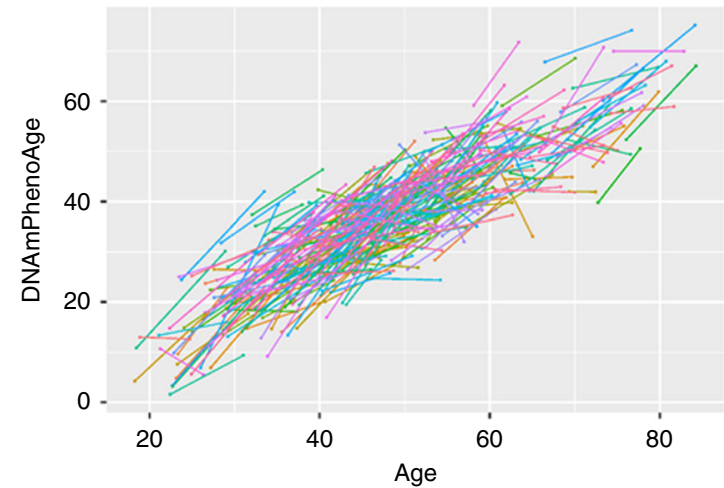

Fig. 2 Spaghetti plots of DNA methylation age versus chronological ages in the longitudinal Enroll-HD data 2. Each spaghetti plot depicts DNAm age versus chronological age, using the Enroll-HD data 2 with two time-points per individual. Each line connects two blood draws from the same individual collected on average 7.9 years apart. The panels report results for different epigenetic clocks a Horvath's pan-tissue clock ${ }^{14}$, b Hannum's blood-based clock $^{15}$, $\mathbf{c}$ the skin and blood clock ${ }^{16}$, and $\mathbf{d}$ and DNAm PhenoAge ${ }^{17}$. The same analysis was not performed on DNAmGrimAge because it is a mortality risk predictor that uses age in its definition.

of epigenetic AgeAccel exhibit nominally significant positive correlations with motor progression (e.g., $r=0.08$, meta-analysis $p=0.016$ for the pan-tissue clock; Supplementary Table 8 and Supplementary Fig. 7).

Human EWAS of HDGEC status. In our EWAS, we related HDGEC status to individual epigenetic markers (CpGs) using Enroll-HD data 1 and Registry-HD, then combined the results using the Stouffer's method. To avoid technical confounding by chip effects in the Enroll-HD data 1, we used the aggregated Enroll-HD data for our EWAS analysis (Supplementary Table 9). The association between HD and individual methylation levels is strongly preserved across the two datasets $(r=0.31$; Supplementary Fig. 8). The Manhattan plot reveals that CpG cg22982173 in exon 1 of the HTT gene on chromosome 4 was most significantly associated with HD status (Stouffer's $p=6.5 \times 10^{-26}$; Fig. $4 \mathrm{a}$ and Supplementary Fig. 9).

We set up a significance threshold at $\alpha=10^{-7}$ to adequately control for false-positive rate in the DNAm array studies ${ }^{20}$. We found 33 genome-wide significant CpGs for HD status (Table 3 and Supplementary Data 1), including cg22982173 in HTT $\left(p=7.0 \times 10^{-26}\right), \operatorname{cg} 26892702$ near DVL2 $(p=5.3 \times$ $\left.10^{-10}\right)$, cg23819669 near DNAJB8 $\left(p=4.2 \times 10^{-9}\right)$, cg19759282 near GPR155 $\left(p=4.4 \times 10^{-9}\right), \operatorname{cg} 16739503$ near RASA3 $(p=6.5 \times$ $\left.10^{-9}\right)$, cg20684718 near MCM10 $\left(p=1.4 \times 10^{-8}\right), \quad$ cg22296756 near HDAC4 $\left(p=6.9 \times 10^{-8}\right)$, and probe ch.3.75336R near MLH1 $\left(p=2.4 \times 10^{-8}\right)$.
HTT methylation across multiple brain regions. We studied HTT cg22982173 using 475 brain methylation samples from our previous study ( $26 \mathrm{HD}$ versus 39 control individuals; "Methods") 6 . Figure 5 lists the bar plots for the seven selected brain regions, with right temporal cortex $(p=0.02$; Fig. $5 \mathrm{e})$ and midbrain $(p=0.046$; Fig. 5h) reaching statistical significance for increased methylation at the HTT cg22982173 locus in HD cases compared to controls. Methylation levels of cg22982173 across all brain regions combined were significantly higher in the HD group (Fig. 5a: $p=5.6 \times 10^{-6}$ and remained significant, $p=1.5 \times 10^{-2}$, after adjusting for intrasubject dependence).

Our top EWAS hit in the blood (in the HTT locus) continues to be associated with HD status in several brain regions (Fig. 5), while the EWAS results in brain tissue are different from those of blood tissue on a global level (Supplementary Fig. 13). Supplementary Figures 11 and 12 report bar plots of cg22982173 stratified by study and tissue types: blood, lymphoblastoid cell, fibroblast, and a total of 14 brain regions. The CpG cg22982173 resides within a region of the genome with a high density of CpGs (i.e., in a $\mathrm{CpG}$ island) and therefore would be expected to have low levels of methylation; however, significant increases in methylation were observed in HD cases.

Both CAG-repeat alleles correlate with human HTT methylation. CAG-repeat lengths were assessed for both long and short alleles (CAG.long and CAG.short) in the human HTT exon 1 locus. We correlated both alleles to human HTT methylation (at cg22982173) using all $N=2164$ human samples. A series of 

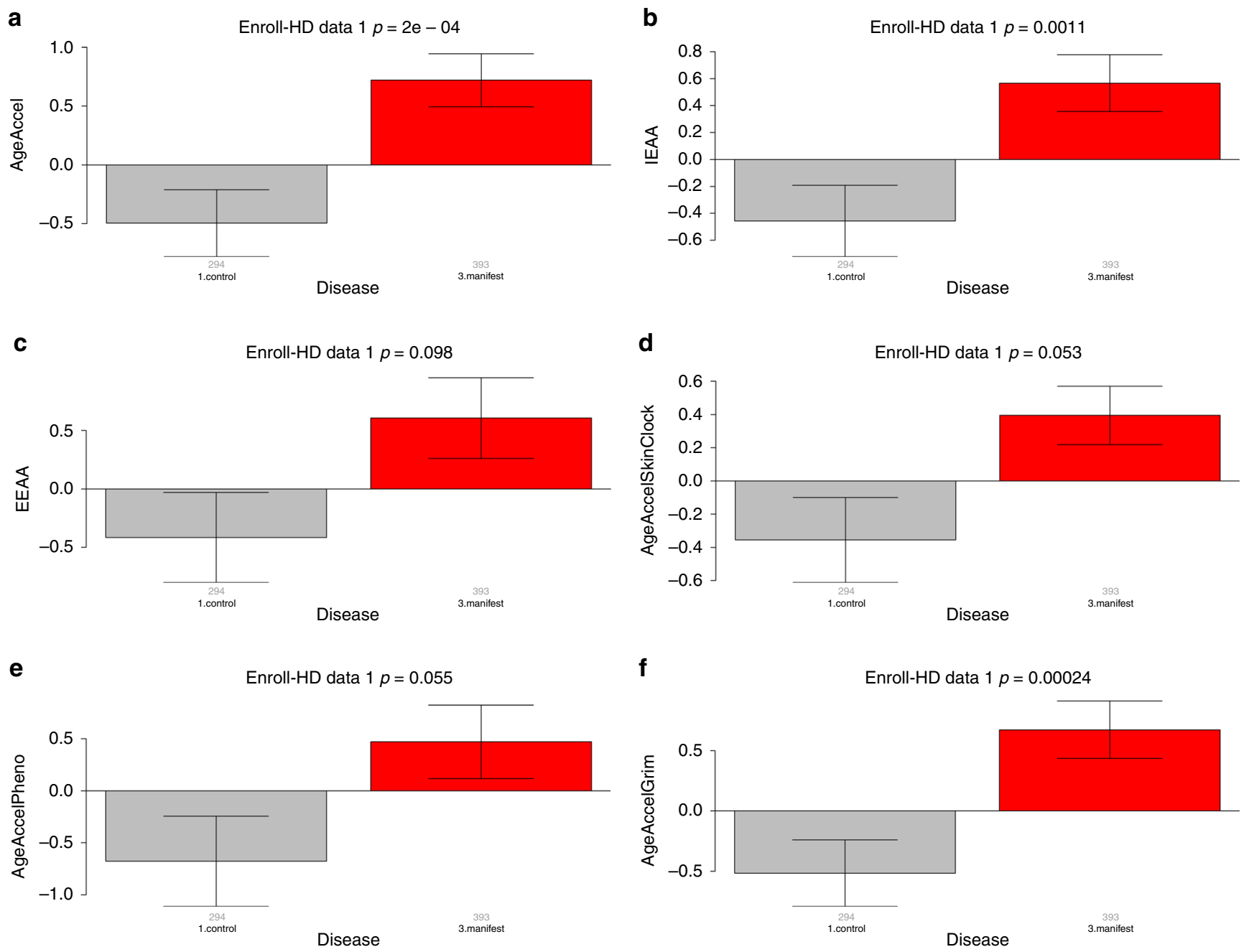

Fig. 3 Epigenetic age acceleration in blood versus manifest HD disease status. Epigenetic age acceleration ( $y$-axis) versus manifest $H D$ status in $N=687$ (294 controls and 393 manifest HD) blood samples from Enroll-HD data 1. Each panel corresponds to a different epigenetic clock. a Age-adjusted DNAm age based on Horvath's pan-tissue clock (AgeAccelerationResidual [AgeAccel] $)^{14}$, $\mathbf{b}$ intrinsic epigenetic age acceleration (IEAA), which is independent of blood cell composition, $\mathbf{c}$ extrinsic epigenetic age acceleration, which is based on Hannum's clock and does depend on blood cell composition ${ }^{15}$, $\mathbf{d}$ ageadjusted DNAm age based on the skin and blood clock (AgeAccelSkinClock) ${ }^{16}$, e age-adjusted DNAm PhenoAge (AgeAccelPheno) ${ }^{17}$, and $\mathbf{f}$ age-adjusted DNAm GrimAge ${ }^{18}$ (AgeAccelGrim). The title of the bar plots reports the nominal two-sided $p$ value from a Kruskal-Wallis test. The $y$-axis of the bar plots depicts the mean and one standard error. Group sizes (i.e., number of individuals per disease category) can be found as small gray numbers underneath each bar.

multivariate regression models were tested to evaluate the joint effect of CAG.long and CAG.short in (i) all, (ii) controls only, and (iii) HD mutation carriers only, respectively ("Methods").

Strikingly, our comprehensive model fitting analysis in different data and cell types demonstrated that both CAG.long and CAG.short were independently associated with HTT methylation (Supplementary Data 2). These significant associations were observed even after restricting the analysis to controls or to $\mathrm{HD}$ mutation carriers, respectively. Interestingly, the product of the two CAG-repeat lengths (CAG.product = CAG. long $\times$ CAG.short) showed the best relationship with HTT methylation according to the Bayesian information criterion (BIC) (Supplementary Data 2). The CAG.product model exhibited the best fit (lowest BIC value) when considering all $N=910$ blood samples from the Enroll-HD data 1 , all $N=376$ blood samples from the Registry-HD, $N=294$ controls from Enroll-HD data 1, N=616 HD mutation carriers from EnrollHD data 1 , the $N=298$ HD mutation carriers from Registry-HD,
$N=80$ mutation carriers from the lymphoblastoid data, and $N=$ 37 mutation carriers from the fibroblast data.

The quadratic term in CAG.long was also significantly related to HTT methylation using polynomial modeling analysis. The multivariate linear models showed that age and sex only have negligible effects on the methylation levels of cg22982173 (Supplementary Data 2).

The moderately high correlation estimates $(r \sim 0.4-0.65)$ between the CAG.product and HTT methylation levels were replicated in several datasets (meta-analysis $p$ value of $9.1 \times$ 10-123; Fig. 6). Significant correlations can be observed even when using only controls (meta-analysis $p=4.5 \times 10^{-8}$ ) or only HD mutation carriers $\left(p=3.9 \times 10^{-27}\right.$; Supplementary Figs. 14 and 15).

The methylation levels of cg22982173 were low both in HD cases and controls: the $\beta$ value (interpreted as the proportion of chromosome methylated at this locus) ranged from 0.015 to 0.063 (Supplementary Fig. 16). 
a

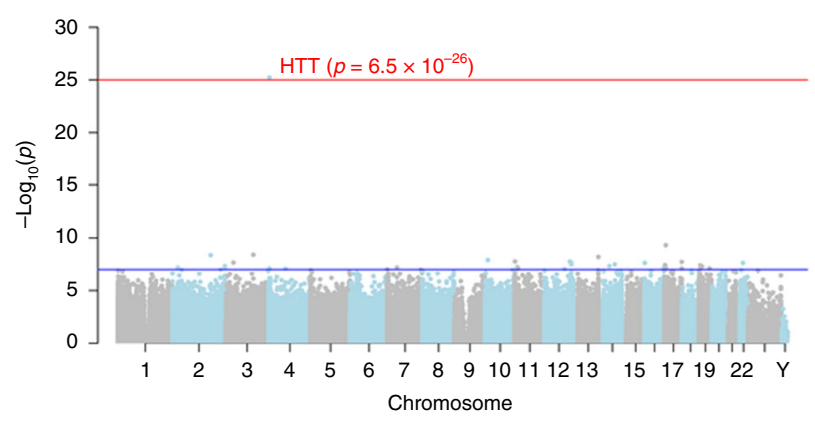

C

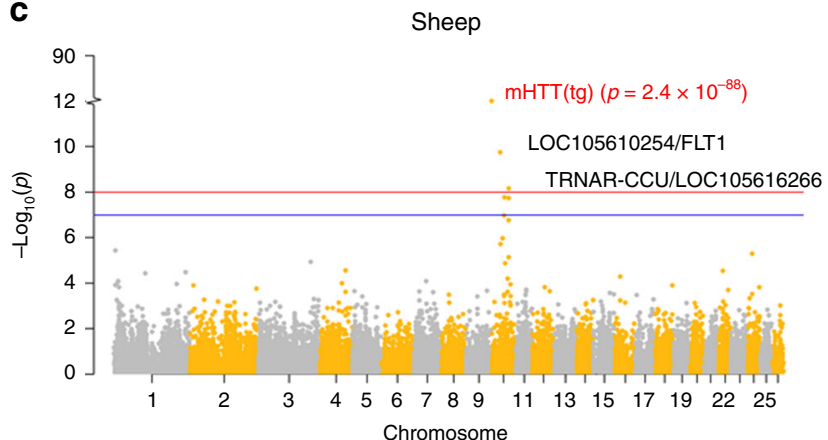

b

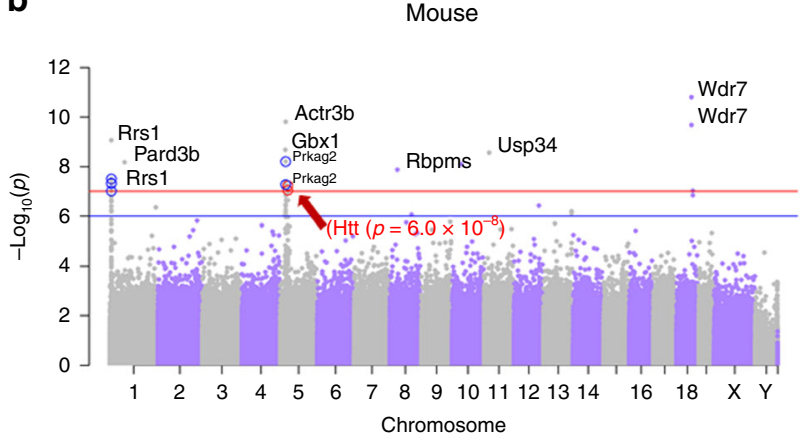

d

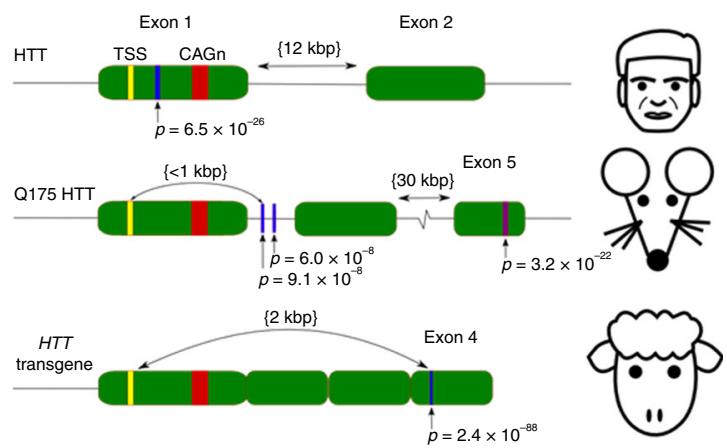

Fig. 4 Epigenome-wide association study (EWAS) of HD status in three species. HDGEC status (carrier versus control) was related to DNA methylation data in three different species: a human blood, b mouse striatum/cerebellum, and $\mathbf{c}$ sheep blood. Each Manhattan plot reports minus log $10^{-t r a n s f o r m e d ~}$ $p$ values ( $y$-axis) versus chromosomal location. Each dot corresponds to a CpG. CpGs near HTT are marked in red. Human HTT is located in chromosome 4. Mouse Q175 knock-in in Htt is located on mouse chromosome 5. Sheep transgenic gene HTT is located on sheep chromosome 10. The blue horizontal lines correspond to genome-wide significance levels for $\mathbf{a}, \mathbf{c}\left(\alpha=1.0 \times 10^{-7}\right)$ and a suggestive significance level for $\mathbf{b}\left(1.0 \times 10^{-6}\right)$; the red horizontal lines correspond to $p<1.0 \times 10^{-32}, p<1.0 \times 10^{-7}$, and $p<1.0 \times 10^{-8}$ for $\mathbf{a}-\mathbf{c}$, respectively; the break in the $y$-axis indicates where the level of significance has been truncated for the purpose of visualization. $\mathbf{b} \mathrm{CpGs}$ marked in blue on chromosome 1 are located in Ryrs1. CpGs marked in red and blue on chromosome 5 are located in Htt and Prkag2, respectively. d The alignment of significant CpGs (blue vertical lines) in the human (hg38), mouse (mm10) and sheep (oviAri4) HTT locus. The significant HTT/Htt CpGs $\left(p<1.0 \times 10^{-7}\right)$ are colored in blue with $p$ values displayed below for human, mouse (RRBS methylation array), and sheep EWAS, respectively. The most significant $\mathrm{CpG}$ found using the mouse methylation array data are colored in magenta.

Human HTT methylation is not confounded by other trinucleotide tandem repeats. It is well known that the HD expansion is overrepresented on a subset of the haplotypes associated with non-disease-associated alleles. Notably, a potential methylationmodifying polymorphism exists in the form of the polymorphic CCG repeat, which lies immediately downstream of the CAG repeat $^{21}$ and tags many of the most common HTT haplotypes.

We evaluated five multivariate linear regression models to examine if the association between HTT cg22982173 and CAG length could possibly arise as an artifact of linkage disequilibrium between the CAG repeat and some other methylation-modifying variants at the HTT locus ("Methods"). Especially, we examined the model (Model 5) that omitted all HD cases that carried an atypical HTT structure, defined as those that do not conform to the "typical" reference alleles $(\mathrm{CAG})_{\mathrm{Q} 1} \mathrm{CAACAGCCGCCA}(\mathrm{CCG})_{\mathrm{P} 2}(\mathrm{CCT})_{2}$ (where Q1 and P2 are numbers of pure CAGs and CCGs, respectively) ${ }^{22}$. We studied 372 individuals from the Registry-HD cohort, which revealed a marked association between CAG- and CCG-repeat lengths in the Registry-HD cohort $\left(r^{2}=0.16, p<2 \times\right.$ $10^{-16}$; Supplementary Fig. 17A). However, several lines of evidence suggest that the most associations we have observed in HTT cg22982173 are not driven by CCG length or other HD-specific haplotype effects, but are a direct product of the CAG length (linear models 1-5 listed in Supplementary Table 10).

Human EWAS of motor progression in manifest HD. To test whether HTT methylation matters for motor progression in manifest HD cases, we performed a meta-analysis that combined three separate EWAS of (adjusted) motor progression using the two Enroll-HD and the Registry-HD datasets, respectively ("Methods"). Interestingly, higher methylation of the HTT locus (cg22982173) was associated with slower HD progression in manifest HD cases according to the motor score $(p=0.05)$, Stroop tests for cognitive function assessment (color naming $p=$ 0.01 , word reading $p=0.07$, and interference test $p=0.06$ ), and functional assessment score $(p=0.009)$.

Our blood EWAS of HD progression in manifest HD cases found three genome-wide significant CpGs: cg26919387 (metaanalysis $p=9.3 \times 10^{-9}$ ) in PEX14, cg12823408 near GRIK4 (meta-analysis $p=3.0 \times 10^{-8}$ ), and cg21497164 in COX4I2 (meta-analysis $p=6.5 \times 10^{-8}$; Fig. 1b, Supplementary Fig. 18, Supplementary Table 11, and Supplementary Data 3 ). The robust correlation (approximate $r=-0.19$ ) indicated that all three CpGs that were hypomethylated were associated with HD motor progression.

The top EWAS hits were not confounded by single-nucleotide polymorphisms (SNPs). CpG measurement can be compromised by neighboring SNPs. We addressed this concern using two approaches. First, we correlated the significant CpGs with SNPs that located near the $\mathrm{CpG}$ probes according to the Illumina annotation file. No significant correlations were observed.

Second, we applied the software Gaphunter ${ }^{23}$ and found only 1 CpG (cg04285477 near Cl4orf43) out of 33 EWAS CpGs associated 
Table 3 Meta-analysis EWAS of HDGEC status in human blood.

\begin{tabular}{|c|c|c|c|c|c|c|c|}
\hline Chr. & CpG & bp & CpG island & Gene & Gene location & Meta $p$ & Meta Z \\
\hline 4 & cg22982173 & $3,076,557$ & Island & HTT & First exon & $6.5 \times 10^{-26}$ & 10.53 \\
\hline 17 & cg26892702 & $7,132,489$ & & DVL2 & Body & $5.3 \times 10^{-10}$ & -6.21 \\
\hline 3 & cg23819669 & $128,187,274$ & & DNAJB8 & TSS1500 & $4.2 \times 10^{-9}$ & -5.88 \\
\hline 2 & cg19759282 & $175,351,649$ & Island & GPR155 & 5'-UTR; 1st exon & $4.4 \times 10^{-9}$ & 5.87 \\
\hline 13 & cg16739503 & $114,862,324$ & S_Shore & RASA3 & Body & $6.5 \times 10^{-9}$ & -5.8 \\
\hline 10 & cg20684718 & $13,248,241$ & & MCM10 & Body & $1.4 \times 10^{-8}$ & -5.68 \\
\hline 12 & cg04605980 & $117,471,700$ & & & & $1.8 \times 10^{-8}$ & -5.63 \\
\hline 11 & cg06993329 & 886,695 & N_Shore & CHID1 & Body & $1.9 \times 10^{-8}$ & -5.63 \\
\hline 17 & cg04683330 & $80,041,239$ & Island & FASN & Body & $1.9 \times 10^{-8}$ & -5.62 \\
\hline 12 & cg16320626 & $121,877,851$ & N_Shelf & $K D M 2 B$ & Body & $2.1 \times 10^{-8}$ & -5.6 \\
\hline 3 & ch.3.753362Ra & $37,048,044$ & & MLH1 & 5'-UTR; body & $2.4 \times 10^{-8}$ & 5.58 \\
\hline 16 & cg09080920 & 928,338 & Island & LMF1 & Body & $2.5 \times 10^{-8}$ & -5.57 \\
\hline 22 & cg14703589 & $31,644,558$ & & LIMK2 & First exon; 5'-UTR; body & $2.6 \times 10^{-8}$ & -5.57 \\
\hline 12 & cg17859634 & $124,798,815$ & Island & FAM101A & Body & $3.4 \times 10^{-8}$ & -5.52 \\
\hline 14 & cg04285477 & $74,194,516$ & & C14orf43 & Body & $3.6 \times 10^{-8}$ & -5.51 \\
\hline 17 & cg23148992 & $3,558,246$ & & CTNS & Body & $4.1 \times 10^{-8}$ & -5.49 \\
\hline 19 & cg08117431 & $5,455,497$ & Island & ZNRF4 & 5'-UTR; 1st exon & $4.2 \times 10^{--8}$ & -5.48 \\
\hline 2 & cg15115604 & $241,069,379$ & Island & MYEOV2 & Body & $5.0 \times 10^{-8}$ & -5.45 \\
\hline 14 & cg10929299 & $50,334,860$ & & & & $5.1 \times 10^{-8}$ & 5.45 \\
\hline 19 & cg10585870 & $14,530,314$ & Island & DDX39 & TSS200 & $5.6 \times 10^{-8}$ & 5.43 \\
\hline 11 & cg15746396 & $13,485,203$ & Island & BTBD10 & TSS1500 & $6.2 \times 10^{-8}$ & 5.41 \\
\hline 7 & cg14640310 & $44,058,913$ & Island & POLR2J4 & TSS200 & $6.9 \times 10^{-8}$ & 5.39 \\
\hline 2 & cg22296756 & $240,305,369$ & & HDAC4 & $5^{\prime}-U T R$ & $6.9 \times 10^{-8}$ & -5.39 \\
\hline 19 & cg11543665 & $8,373,294$ & Island & CD320 & TSS201 & $6.9 \times 10^{-8}$ & 5.39 \\
\hline 2 & cg05054944 & $25,856,333$ & & DTNB & Body & $7.2 \times 10^{-8}$ & -5.39 \\
\hline 16 & cg00284420 & $87,311,948$ & & & & $7.5 \times 10^{-8}$ & -5.38 \\
\hline 17 & cg24965497 & $7,227,181$ & N_Shore & NEURL4 & Body & $7.6 \times 10^{-8}$ & -5.38 \\
\hline 4 & cg13731523 & $3,047,190$ & S_Shelf & & & $8.0 \times 10^{-8}$ & -5.37 \\
\hline 17 & cg14517001 & $80,179,931$ & Island & & & $8.2 \times 10^{-8}$ & -5.36 \\
\hline 19 & cg20635595 & $48,281,627$ & Island & SEPW1 & TSS1500 & $8.4 \times 10^{-8}$ & 5.36 \\
\hline 11 & cg16356630 & $14,683,035$ & & PDE3B & Body & $8.7 \times 10^{-8}$ & -5.35 \\
\hline 4 & cg01200289 & $77,227,751$ & Island & STBD1 & First exon; 5'-UTR & $9.0 \times 10^{-8}$ & 5.35 \\
\hline 12 & cg11881599 & $92,814,084$ & & CLLU1OS & 3'-UTR; TSS1500 & $9.2 \times 10^{-8}$ & 5.34 \\
\hline
\end{tabular}

The table reports 33 genome-wide significant $\mathrm{CpGs}$ (meta-analysis $p$ value $<10^{-7}$ ). A positive (negative) value of the meta-analysis $Z$ statistic indicates that the $\mathrm{CpG}$ is hypermethylated (hypomethylated) in human HDGECs. In this case-control study, we did not distinguish pre-manifest from manifest HD. The $Z$ statistic is based on Stouffer's meta-analysis method across the aggregated Enroll-HD samples and the Registry-HD data. Columns report the chromosome, CpG, position (hg19 assembly), the relative location to the nearest $\mathrm{CpG}$ island, nearest gene, location of the CpG with respect to the gene, meta-analysis (unadjusted, two-sided) $p$ value, and $Z$ statistic.

aThe unusual probe name "ch.3.753362R" reflects a $\mathrm{CpH}$ probe (CA, CC, CT) as opposed to a CpG probe, which targets the reverse strand on chromosome 3 (hg19 coordinate 753362 ).

with HD disease status exhibited weak evidence of confounding (i.e., a clustering pattern; "Methods"). None of the three CpGs (top hits) associated with motor progression showed evidence of confounding.

EWAS of motor progression was not confounded by treatment. Tetrabenazine, a medication for symptomatic treatment of chorea, was used by $\sim 23 \%$ of HD patients in Enroll-HD. Treated and untreated HD patients did not differ in terms of age, but treated patients exhibited a higher CAP score $(p=0.01$ in EnrollHD data 1 and $p=8.2 \times 10^{-8}$ in Enroll-HD data 2$)$ and a higher motor score $\left(p=3.2 \times 10^{-4}\right.$ in Enroll-HD data 1 and $p=2.1 \times$ $10^{-9}$ in Enroll-HD data 2; Supplementary Figs. 19 and 20). Tetrabenazine was not significantly associated with any of the three motor progression-associated CpGs as depicted in Supplementary Fig. 21. Furthermore, no CpGs on the Illumina array were associated with tetrabenazine treatment at a genome-wide significance level of $p=1.0 \times 10^{-7}$ (Supplementary Fig. 22). Overall, these results demonstrate that the EWAS of HD progression is not confounded by tetrabenazine treatment.

Methylation data from $\mathbf{H t t}$ knock-in mice (Q175). We generated two types of DNAm datasets (RRBS and custom methylation array) from a heterozygous (HET) Htt knock-in (KI) mouse model (Q175 versus Q20 CAG repeats or wild-type mice; "Methods").

We conducted EWAS of mutant HTT gene (Q20 versus Q175) across the striatum and cerebellum in RRBS data. Focusing on CpGs in the murine Htt locus revealed a moderately strong correlation between the striatum and the cerebellum $(r=0.55$ and $p=5.3 \times 10^{-11}$; Supplementary Fig. 23). Strikingly, $\mathrm{CpG}$ sites falling within the $H t t$ locus are among the top EWAS results for Q175 status in murine striatum and cerebellum (Supplementary Fig. 24). This finding is also illustrated by our meta-analysis EWAS across the striatum and cerebellum (Fig. 4b). Our EWAS identified two genome-wide significant $\mathrm{CpGs}$ in $\mathrm{Htt}$ region, chr5:34762314 (meta-analysis $p=6.0 \times 10^{-8}$ ) and chr5:34762241 $\left(p=9.2 \times 10^{-8}\right)$ located between exons 1 and 2 (Fig. $4 \mathrm{~b}, \mathrm{~d}$ and Supplementary Data 1 ). These CpGs were located $\sim 1 \mathrm{~kb}$ in the $3^{\prime}$ direction of HTT exon1 (Fig. 4d), both were hypermethylated in the Q175 group.

Analyzing the same brain regions from another group of mice using our custom array platform revealed another highly significant $\mathrm{CpG}$ to be hypomethylated in the Q175 group (probe cg12389415 on the custom array, mouse chr5:34795955) also within the Htt locus (meta-analysis $p=3.2 \times 10^{-22}$; Supplementary Fig. 25). Integrating the EWAS results across all the available tissue types: the two brain regions, blood, cortex, and liver, increased the statistical significance of the association (Stouffer meta-analysis $p=6.2 \times 10^{-45}$ ). The $H t t$ gene locus was the top 
a

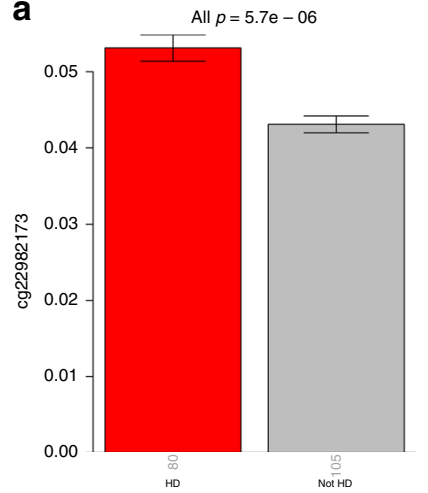

e

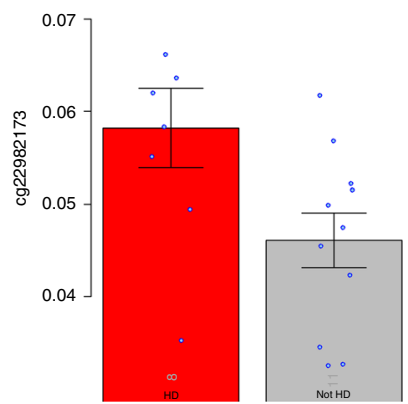

b

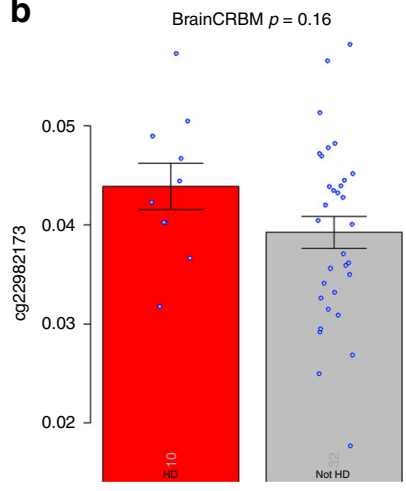

f

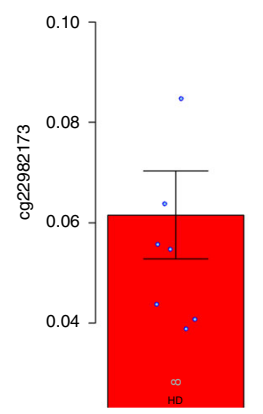

C

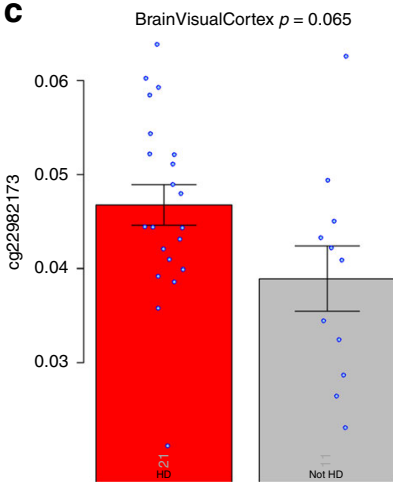

g

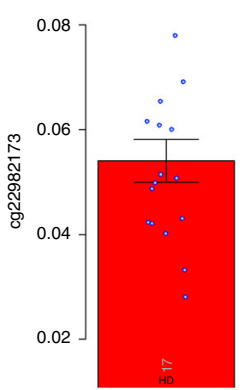

d

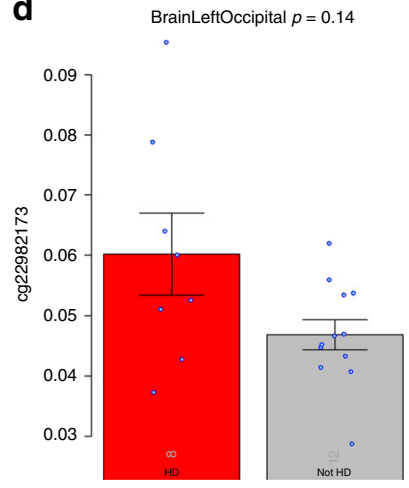

h

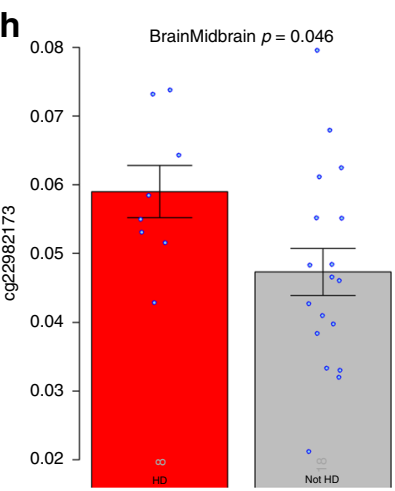

Fig. 5 Human HTT methylation in different brain regions. The figure depicts the bar plots for the association of HD disease status with HTT cg22982173 in different brain regions ( $N=67$ from $26 \mathrm{HD}$ versus 39 control individuals). We used $n=185$ brain tissues ( $80 \mathrm{HD}$ versus 105 controls) from seven brain regions: a bar plot based on all the seven brain regions combined. The results for a remain significant $(p=0.015)$ after adjusting for intra-individual correlations using a linear mixed-effects model. b-h Bar plots for each individual region: cerebellum (CRBM), visual cortex, left occipital, right temporal cortex, left frontal cortex, caudate nucleus, and midbrain, respectively. The blue dots correspond to individual observations that underlie the respective mean values. The $y$-axis of the bar plots depicts the mean of one standard error. The numbers of individuals per group are reported as gray numbers in each bar. The heading of each panel reports a nominal (unadjusted) two-sided $p$ value (Kruskal-Wallis test).

EWAS hit in all five tissues profiled on the custom methylation array (Supplementary Data 1).

Ovine EWAS of HD status. In addition to mice, we also studied a transgenic model of sheep with a human HTT cDNA transgenic sequence $^{24-28}$. The transgene is integrated into a different location than the endogenous sheep HTT gene. Using a custom methylation array, we generated blood methylation data from 168 sheep: 84 HD transgenic sheep and age-matched controls; "Methods"). EWAS of HD transgenic status identified five genome-wide significant $\left(p<1.0 \times 10^{-7}\right)$ CpGs located on chromosome 10 including one $\mathrm{CpG}$ near the HTT transgene (Fig. 4c, according to oviAri4 assembly coordinates 1826-1903 bp). The most significant association was the CpG located in exon 4 of the HTT transgene with a striking $p$ value of $2.4 \times 10^{-88}$, followed by another CPG in the FLT1 gene $\left(p=1.8 \times 10^{-10}\right.$; Supplementary Data 1).

Only CpGs close to the CAG expansion are hypermethylated in HD. A comparison of the significant CpGs in the HTT locus across three species, human, mouse and sheep (Fig. 4d), revealed that significant $\mathrm{CpGs}$ for HD status are located within $2 \mathrm{~kb}$ of the CAG expansion in exon 1 of HTT/Htt. The comparison between human and sheep highlighted that the proximity between a $\mathrm{CpG}$ dinucleotide and the location of the CAG expansion in either the endogenous HTT or transgenic HTT (typically $<2 \mathrm{~kb}$ ) plays an important role (Fig. 4d). This is based on the following observations: (a) all genome-wide significant CpGs near the HTT locus are located within $2 \mathrm{~kb}$ of the CAG expansion, (b) unlike in humans, HTT exon 4 is close to the site of the CAG expansion in the sheep because the HTT transgene is a cDNA without any intronic genomic DNA, (c) unlike in humans, methylation at a specific CpG in exon 4 of an HTT cDNA gene fragment relates strongly to HD transgenic gene status in blood samples from the sheep (Supplementary Data 1).

Next, we investigated if HD patients tended to gain methylation in associated HTT CpGs. Interrogating the genomic regions surrounding HTT exon 1, all four CpGs were significantly hypermethylated in HD patients (Supplementary Data 4). Interestingly, all the 27 significant CpGs in Htt (meta-analysis $p<0.05$ ) also exhibited the same hypermethylation pattern in Q175 mice (Supplementary Data 4).

Enrichment analysis of EWAS results. To gain insights on the biological function associated with $\mathrm{HD}$ related $\mathrm{CpGs}$, we used two different approaches for carrying out functional enrichment analysis: (1) the anRichment approach under HDinHD ${ }^{29}$ and (2) GOMETH enrichment analysis, which takes into account the different number of probes per gene present on the Illumina $450 \mathrm{k}$ array $^{30}$. We studied the top $1000 \mathrm{CpG}$ that were hypomethylated in $\mathrm{HD}$ and another set of top $1000 \mathrm{CpGs}$ that were hypermethylated in HD using the anRichment analysis (Supplementary Tables 12 and 13). The top 1000 hypomethylated CpGs were adjacent to genes (hypergeometric $p=3.6 \times 10^{-13}$ ) implicated by a protein-protein interaction network analysis of polyglutamine disorders (Supplementary Table 14). The most significant enrichment according to the GOMETH analysis involved the 
a

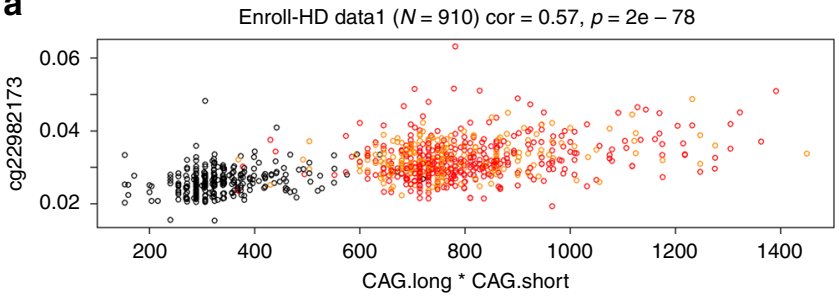

b
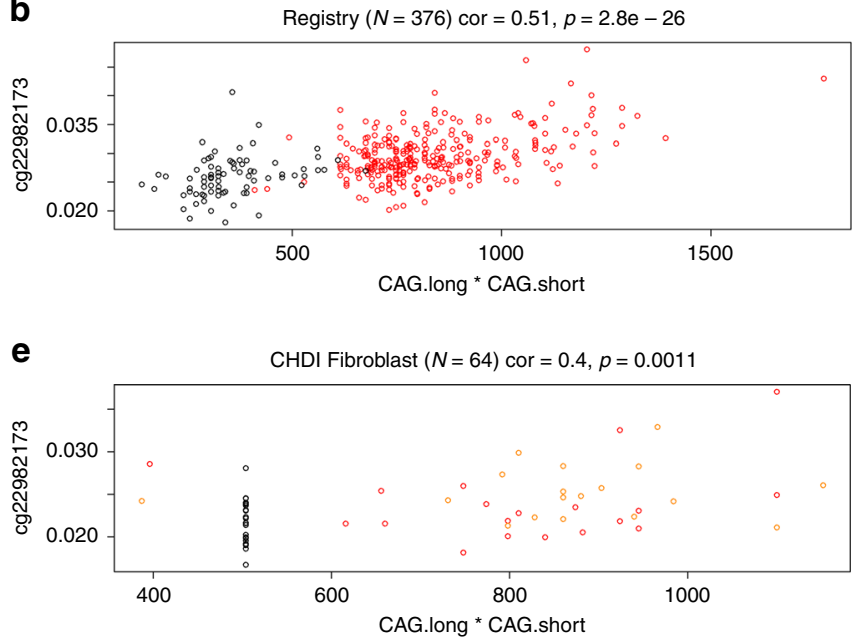

b

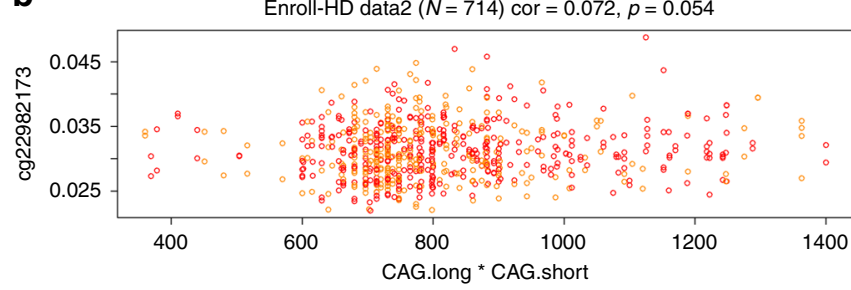

d

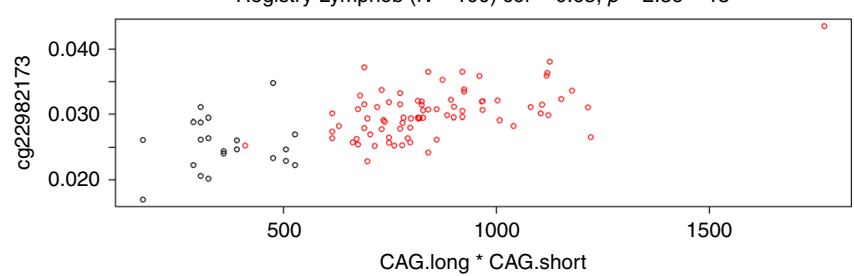

Fig. 6 Human HTT methylation versus the product of CAG lengths. The product of the two CAG alleles (CAG.product $=$ CAG.long $\times$ CAG.short) correlated with DNA methylation levels of the human HTT cg22982173 locus in a blood samples from Enroll-HD data 1, b blood samples from Enroll-HD data 2, c blood samples from the Registry-HD study, d lymphoblastoid lines from the Registry-HD study, and e fibroblast samples from the MTM study. The title of each panel reports the Pearson's correlation coefficient and corresponding unadjusted $p$ value. Fixed-effects meta-analysis (weighted by inverse variance) produced a meta-analysis $p$ value of $9.1 \times 10^{-123}$. Points in the scatter plots are colored by HD status: red $=$ manifest $\mathrm{HD}$, orange $=$ pre-manifest $\mathrm{HD}$, and black $=$ control. Each plot reports a Pearson's correlation coefficient and corresponding $p$ value.

overlap between the top 1000 hypermethylated CpGs and RNA binding ( $p=3.4 \times 10^{-6}$; Supplementary Table 15$)$.

We carried out an enrichment analysis of CpGs associated with HD progression using the same two approaches. The anRichment analysis identified the HD progression related genes enriched in several known HD relevant gene sets (Supplementary Table 16) and the GOMETH analysis identified gene sets enriched in immune cell activation (Supplementary Table 17).

\section{Discussion}

This is by far the largest DNAm study of HD to date. We generated large methylation datasets from seven sources of DNA across three species (human: 2000 blood, 100 lymphoblastoid cells, and 64 fibroblasts; mouse: 32 striatum, 32 cerebellum, 16 liver, 16 cortex, and 16 blood; sheep: 168 in the blood).

One of our major findings is that manifest (but not premanifest) HD cases exhibit accelerated epigenetic aging effects in blood, which is consistent with the epigenetic AgeAccel observed in various brain regions ${ }^{6}$. HD progression in manifest individuals was only weakly linked to epigenetic AgeAccel in blood.

A second major finding of our study was that EWAS (as opposed to GWAS) of the HTT mutation status consistently found CpGs in the HTT locus in all species and tissues. If nothing was known about the genetics of HD, then our human blood methylation data would have implicated the pertinent disease gene: a very significant CpG probe located in HTT exon 1 . The HTT methylation effect was validated across seven sources of DNA (blood, lymphoblastoid cells, fibroblasts, striatum, cerebellum, cortex, and liver) and across three species. Our most significant human HTT cg22982173 was neither confounded by neighboring trinucleotide tandem repeats nor dominated by atypical HTT structure. We also analyzed custom methylation array data (providing $>1000 \times$ coverage per $\mathrm{CpG}$ ) to address concerns surrounding low sequence coverage (minimum $15 \times$ coverage) in the mouse RRBS data ${ }^{31}$. Both mouse RRBS and custom methylation array data implicated the $\mathrm{Htt}$ gene. A comparison of the significant CpGs in the HTT/Htt locus across three species-human, mouse, and sheep-revealed that significant CpGs for HD status were located in the vicinity of the CAG expansion in exon 1 of HTT/Htt. The methylation levels of cg22982173 exhibited only weak (but nominally significant) negative correlations with measures of motor progression and other clinical assessments (e.g., Stroop test) in manifest HD after adjusting CAG expansion, and other potential confounders. An intriguing and yet unexplained finding, based on our comprehensive model fitting analysis of the most significant CpG cg22982173 in the human HTT locus, was that the methylation level at this locus correlated with the allelic product of CAG lengths in both cases and controls, inferring that methylation at this locus was related to the length of the non-mutated allele as well. Our analysis of HTT methylation in human postmortem brain samples was consistent with that observed in blood samples.

Abundant caution needs to be exercised in linking Htt exon1 methylation to expression levels of the mutant HTT/Htt gene, since CpGs often have only weak effects on neighboring gene expression levels ${ }^{32}$. However, it is interesting that at least in the murine $\mathrm{Htt}$ allelic series KI mouse model, there is a robust $\mathrm{Htt}$ CAG-length-dependent reduction of mutant $H t t$ RNA and protein levels in the striatum ${ }^{29}$. Caution is also warranted when interpreting the relationship between transgene expression and CpG methylation in the transgenic sheep model for the following 
reasons. When exogenous DNA is inserted into a host genome in order to generate a transgenic cell or animal model, copy number, integration site, and transgene composition (e.g., the inclusion of exogenous viral or bacterial sequences) could all significantly influence the status of transgene methylation and expression ${ }^{33,34}$. Moreover, transgenes can be considerably affected by the flanking genomic DNA sequences at its random integration site, hence susceptible to position effect variegation including gene silencing 35,36 .

Our finding on HTT/Htt locus methylation, especially identifying that the most significant HD-associated methylation sites were in close proximity to the repeat expansion mutation itself, may have general implications for understanding the role of epigenetics in repeat expansion disorders. First, similar findings of pathologically relevant abnormal DNAm in the proximity of expanded pathogenic repeats have been observed in other disorders such as fragile $\mathrm{X}$ syndrome, myotonic dystrophy type I, and Friedreich's ataxia ${ }^{37-39}$. Second, a recent study showed 22 disease-associated tandem repeats, including those found within genes: FMR1 (gene mutated in fragile X), frataxin, HTT, ataxin 1, and ataxin 3 were located in chromatin domain boundaries enriched with an ultra-high density of $\mathrm{CpG}$ islands and bound by methylation-sensitive chromatin factor $C T C F^{40}$.

Another important area of investigation is whether methylation changes on HTT exon 1 have any impact on the somatic instability of the mutant CAG repeat itself. Recent large-scale human genetic studies revealed variants in mismatch repair enzymes (e.g., $M L H 1, M S H 2, M S H 3)$, which are known to modify the germline and somatic instability of expanded HTT CAG repeats in KI mouse models $s^{41}$, act as modifiers of the age of motor symptom onset in $\mathrm{HD}^{5}$.

Our EWAS of HDGEC status in human blood identified 33 loci at a genome-wide significance level. Besides HTT itself, the EWAS revealed the following genes known to modify mutant HTT aggregation or toxicities. DNAJB8 is a member of the DNAJ (Hsp40) chaperone family and inhibits polyglutamine mutant HTT aggregation and amyloid fibril formation ${ }^{42,43}$. HDAC4 binds to mutant $H T T$ in a polyglutamine length-dependent manner and is localized to the cytoplasmic inclusions in striatal neurons in HD mouse models, and genetic reduction of murine $\mathrm{Hdac} 4$ reduces cytoplasmic mutant $\mathrm{Htt}$ aggregation and restores cortico-striatal synaptic transmission in HdhQ150 KI mice ${ }^{44}$.

Another clinically relevant finding determined by blood EWAS of $\mathrm{HD}$ motor progression was that epigenetic modifiers were linked to symptom progression in HD. Our analysis focused on progression of motor symptoms in manifest $\mathrm{HD}$ cases discovered three CpGs: cg26919387 near PEX14 $\left(p=9.3 \times 10^{-9}\right)$, cg12823408 near GRIK4 $\left(p=3.0 \times 10^{-8}\right)$, and cg21497164 in COX4I2 $(p=$ $\left.6.5 \times 10^{-8}\right)$. These findings were not confounded by medications used for treating movement disorders (tetrabenazine). Arguably, the most interesting gene identified was GRIK4, which is selectively expressed in the cortex and striatum. Studies of mice deficient in Grik4 or elevated Grik4 gene dosage have demonstrated the critical role of this molecule in sensory motor gating and protection against excitatory neurotoxicity ${ }^{45}$.

While our EWAS findings in blood were different to those observed in a previous analysis of brain tissue using a limited brain methylation dataset ${ }^{6}$, we found that several brain regions exhibited hypermethylation of the HTT cg22982173 locus in HD cases. In our two independent murine studies, we observed genome-wide significant hyper- and hypomethylation at the $\mathrm{Htt}$ locus in the striatum and cerebellum of Q175 KI mice. Furthermore, our top EWAS hit inside the HTT locus replicated in human lymphoblastoid cells and in fibroblasts.

Overall, our study provides new insights and directions of HD research in the areas of accelerated epigenetic aging, the role of
HTT locus methylation and HD biology, and other methylation sites that may act as epigenetic modifiers that modulate HD progression.

\section{Methods}

Data sets. HD mutation carriers did not differ significantly from controls in terms of chronological age. However, pre-manifest HD samples tended to be significantly younger than manifest HD samples. Further, the study sample ascertainment induced a strong negative correlation between CAG length and age at enrollment $\left(r=-0.5, p=2.4 \times 10^{-40}\right.$ in Enroll-HD). Thus, manifest HD status (or equivalently pre-manifest HD status) was confounded by chronological age in our data Unfortunately, we observed a strong association between HD disease status and Illumina chip ID in the Enroll-HD study (but not in the Registry-HD study). Most Illumina chips contained 12 manifest HD samples or 12 pre-manifest HD samples or 12 controls. Since chip effects probably confound the relationship between HD status and DNAm levels, we removed the chip effects from the DNAm data by averaging the DNAm levels of each CpG across the 12 samples on a given chip. The HD status of a chip corresponded to the shared HD status of the 12 samples. By replacing individual level data by "aggregated Enroll-HD data," we effectively removed chip effects at the cost of reduced sample sizes (from $N=910$ Enroll-HD samples to 76 corresponding chips). Illumina chips were not confounded by HD status in the Registry-HD study.

Enroll-HD: a prospective registry study in a global HD cohort. We obtained phenotypic data (demographic data and clinical assessments) from the longitudinal Enroll-HD study based on 15,203 individuals. Only a subset of these 15,203 individuals was profiled on the DNAm array: (1) Enroll-HD data 1 were based on a single buffy coat sample from $N=910$ individuals (controls, pre-manifest $\mathrm{HD}$, and manifest HD) from Enroll-HD data 1. The Enroll-HD data 2 involved two buffy coat samples (from two separate blood draws) from 368 HTT mutation carriers. The first blood sample from each individual was on average collected 7.9 years before the second sample. We removed 11 of the 368 individuals from the analysis because (a) two individuals were severe outliers according to our epigenetic clock analysis and nine individuals already appeared in the Enroll-HD data 1. Thus, we only studied 357 individuals from the Enroll-HD data 2 study in our analysis.

Enroll-HD is a longitudinal, observational, multinational study that will integrate two existing HD registries, Registry-HD in Europe and COHORT in North America and Australia, while also expanding to include sites in Latin America and Asia. ClinicalTrials.gov Identifier: NCT01574053 (Principal Investigator: Bernhard G. Landwehrmeyer and Study Director: Joseph Giuliano).

With indefinite and ongoing annual assessments, the goal of Enroll-HD is to build a large and rich database of longitudinal clinical information and biospecimens. This database will serve as a basis for future studies aimed at developing tools and biomarkers for progression and prognosis, identifying clinically relevant phenotypic characteristics, and establishing clearly defined endpoints for interventional studies. Core datasets are collected annually on all research participants as part of this multicenter longitudinal observational study of HD. Data are monitored for quality and accuracy using a risk-based monitoring approach. All sites are required to obtain and maintain local Ethics Committee approvals.

The primary objective of Enroll-HD is to develop a comprehensive repository of prospective and systematically collected clinical research data (demography, clinical features, family history, genetic characteristics) and biological specimens (blood) from individuals with manifest $\mathrm{HD}$, unaffected individuals known to carry the HD mutation or at risk of carrying the HD mutation, and control research participants (e.g., spouses, siblings, or offspring of HD mutation carriers known not to carry the HD mutation). Enroll-HD is conceived as a broad-based and long-term project to maximize the efficiencies of non-clinical research and participation in clinical research. With over 200 sites in roughly 30 countries, Enroll-HD is the largest database available for HD researchers. Our Enroll-HD data 1 involved blood samples from Northern America (81\%), Europe (12.8\%), Latin America (1.1\%), and Oceania (5.1\%). All individuals were 18 years or older. Both sexes were eligible for the study as were healthy volunteers. A non-probability sampling method was used.

Informed consent from the potential participant or legal representative is a prerequisite for study participation. Participants' IRB consent is an unconditional prerequisite for patient participation in the Enroll-HD, approved by the Scientific Review Committee [https://enroll-hd.org/for-committees/]. More details of the ethics oversight are listed in Enroll-HD protocol [https://www.enroll-hd.org/ enrollhd_documents/Enroll-HD-Protocol-1.0.pdf].

Below, we describe the study population from Enroll-HD. Patients with HD and their family members are recruited from speciality clinics (Human Genetics, Neurology, Psychiatry) that advise and treat people affected by HD. In addition, in some areas, community clinics and neurologists who see HD patients will recruit participants for this study. More details for the study population including inclusion and exclusion criteria are listed in Supplementary Note 2.

Registry-HD data. The European Huntington's Disease Network (EHDN) provided us with whole DNA samples from the Registry-HD core research project. We analyzed 376 samples from whole blood and 100 samples from lymphoblastoid cell 
lines. Registry-HD is a multi-center, multinational observational study with no experimental treatment. It forms part of the Huntington Project, a worldwide collaboration dedicated to finding treatments that make a difference for $\mathrm{HD}$. Registry-HD is sponsored by the High Q Foundation, a non-profit organization that supports a variety of research projects seeking to find treatments for $\mathrm{HD}$. The study description of Registry-HD is listed in NIH ClinicalTrial database [https:// clinicaltrials.gov/ct2/show/NCT01590589]. Participants IRB consent was obtained at the time of the potential participant's visit an explanation of the study, approved by the Scientific and Bioethical Advisory Committee of the EHDN. Details of the consent are listed the section of "Participant informed consent" of the study protocol [http://www.ehdn.org/wp-content/uploads/2018/06/registry-protocol-2.0. pdf].

Human fibroblasts. We obtained $N=64$ fibroblast samples from the CHDI Multiple Tissue Monitoring (MTM) study. The study involved 37 HD mutation carriers and 27 age-matched controls. The mean age was 42.7 years and the proportion of males was $45 \%$. DNAm arrays were profiled in fibroblast tissues. The mean CAG lengths in the HD mutation group were 44 and 19 for the long and short allele, respectively. The CAG lengths were not available in the control group and were set to 28 and 18 for the long and short allele, respectively. The data are described in Table 1.

DNAm data. DNA was extracted from the Registry-HD and the Enroll-HD samples using the Miller salting out procedure and the QIAGEN QIAamp DNA Midi Kit. DNA was eluted in Buffer AE (a TE buffer). Bisulfite conversion was performed using the Zymo EZ DNA Methylation Kit (Zymo Research, Orange, CA, USA) as well as subsequent hybridization to the Illumina DNAm array, and scanning (iScan, Illumina) were performed according to the manufacturer's protocols by applying standard settings. DNAm array data were profiled in HumanMethylation450 Bead Chip for Enroll-HD data 1 and Registry-HD, and profiled on the EPIC array (also known as 850k array) for Enroll-HD data 2. DNAm levels ( $\beta$ values) were determined by calculating the ratio of intensities between methylated (signal A) and un-methylated (signal B) sites. Specifically, the $\beta$ value was calculated from the intensity of the methylated ( $M$ corresponding to signal $A$ ) and unmethylated (U corresponding to signal B) sites, as the ratio of fluorescent signals $\beta=\operatorname{Max}(\mathrm{M}, 0) /[\operatorname{Max}(\mathrm{M}, 0)+\operatorname{Max}(\mathrm{U}, 0)+100]$. Thus, $\beta$ values range from 0 (completely un-methylated) to 1 (completely methylated). We used the "noob" background normalization method ${ }^{46}$ to account for technical variation in background fluorescence signal. The noob approach capitalizes on a new use for the Infinium I design bead types to measure nonspecific fluorescence in the color channel opposite of their design (Cy3/Cy5).

DNAm age and epigenetic clock. DNAm levels give rise to particularly promising biomarkers of aging since chronological age (i.e., the calendar years that have passed since birth) has a profound effect on DNAm levels in most human tissues and cell types. To study the association of epigenetic AgeAccel with HD, we used (1) Horvath's pan-tissue DNAm age on the basis of $353 \mathrm{CpGs}^{14}$, (2) Hannum's DNAm age on the basis of $71 \mathrm{CpGs}^{15}$, (3) DNAmAgeSkinClock on the basis of 391 $\mathrm{CpGs}^{16}$, (4) DNAm PhenoAge on the basis of $513 \mathrm{CpGs}^{17}$, and (5) DNAm GrimAge on the basis of $1030 \mathrm{CpGs}^{18}$. The first three DNAm ages were developed as a measure of chronological age, while the last two were developed as a measure of mortality risk. However, all the biomarkers are associated with mortality and morbidity ${ }^{17,18,47}$

All of the DNAm age models were established through elastic net regression models. For example, the pan-tissue clock is based on methylation levels of 353 dinucleotide markers known as cytosine phosphate guanines or $\mathrm{CpGs}^{14}$. The weighted average of these 353 epigenetic markers gives rise to an estimate of tissue age (in units of years), which is referred to as "DNAm age" or as "epigenetic age." The pan-tissue epigenetic clock method applies to any tissue or cell type that contains DNA (with the exception of sperm), including individual cell types (helper T cells, neurons, glial cells) and complex tissues and organs (blood, brain, bone, breast, kidney, liver, lung $)^{14}$

To study the association between the DNAm-based biomarkers and HD, we used a measure of epigenetic AgeAccel that has been widely used in our previous studies of aging. We regressed DNAm age on chronological age using a linear regression model and defined AgeAccel as the corresponding raw residuals. By definition, AgeAccel has no linear dependence on chronological age (correlation $r=0$ ). Thus, a positive (or negative) value of AgeAccel indicates that the DNAm Age is higher (or lower) than expected based on age. In an analogous manner, we defined the acceleration measures, AgeAccelSkinClock, AgeAccelPheno, and AgeAccelGrim, based on DNAmAgeSkinClock, DNAmAgePheno, and DNAmGrimAge, respectively. We further examined two widely used acceleration measures: IEAA derived from Horvath's pan-tissue DNAm Age, which is independent of age-related changes in blood cell composition, and extrinsic epigenetic age acceleration based on Hannum's DNAm Age, which up-weights the contribution of blood cell count measure.

Mathematical details and software tutorials for the epigenetic clock can be found in the Supplements of the relevant publications ${ }^{14}$. An online age calculator can be found at our webpage [https://dnamage.genetics.ucla.edu].
EWAS of HD disease status. We adjusted the CpG data for sex using a linear model, then related each $\mathrm{CpG}$ to HD status using the robust biweight midcorrelation. We used the "estlambda" function in the GenABEL R package to calculate the inflation factors ${ }^{48}$.

We stratified our EWAS analysis by pre-manifest/manifest HD disease status because each stratum lends itself for addressing different biological questions: studying time to $\mathrm{HD}$ onset may involve different pathways from those that determine motor progression in manifest HD samples. Because sex can have an effect on DNAm levels, we adjusted the DNAm levels for sex by forming residuals. Since HD germline mutation status was not related to chronological age, there was no need to adjust for chronological age.

Our analysis methods make extensive use of meta-analysis. A simple yet powerful meta-analysis method, known as Stouffer's method, relies on combining the $Z$ statistics from individual datasets (the two blood datasets (aggregated EnrollHD and the Registry-HD study). Specifically, for each CpG $i$ and dataset $a$, one obtains a $Z$ statistic $Z_{i a}$, for example, by the inverse normal transformation of the $p$ value. Next, a meta-analysis $Z_{i}$ statistic for each CpG is calculated as

$$
Z_{i}=\frac{1}{\sqrt{N_{\text {sets }}}} \sum_{a=1}^{N_{\text {sets }}} Z_{\text {ia }}
$$

The meta-analysis statistic $Z_{i}$ is approximately normally distributed with mean 0 and variance 1 ; the corresponding $p$ value is then calculated using the normal distribution.

Human brain methylation data in multiple regions. We studied the association of HD with brain methylation levels of HTT CpG using our previous study data ${ }^{6}$. Postmortem brain samples from HD $(N=26)$ and non-HD $(N=39$ including 18 Alzheimer's disease cases and 21 neurologically normal controls) were collected at UCLA and University of Auckland. DNAm arrays were profiled in following brain regions: caudate nucleus ( $N=29$ arrays), cingulate gyrus $(N=33)$, cerebellum $(N=42)$, hippocampus $(N=33)$, parietal cortex $(N=64)$, frontal lobe $(N=70)$, occipital cortex $(N=43)$, temporal cortex $(N=37)$, midbrain $(N=26)$, motor cortex $(N=33)$, sensory cortex $(N=33)$, and visual cortex $(N=32)$. In our association analysis for HD disease status, we removed the samples from left temporal cortex $(N=0$ for HD), right frontal cortex $(N=0$ for $\mathrm{HD})$, and right occipital regions ( $N=2$ for HD), yielding a total of 14 brain sub-regions available for our analysis.

Allelic effects of CAG length alleles on HTT CpG. We studied the joint effect of CAG.long and CAG.short on human HTT methylation (at cg22982173) in N= 1807 individuals (2164 samples) across five datasets: Enroll data 1 (blood samples), Enroll data 2 (two blood samples for each individual), Registry-HD blood samples, Registry-HD lymphoblastoid cells, and CHDI fibroblast. We tested eight multivariate regression models to evaluate the joint effect of CAG.long, CAG.short, and a quadratic term in CAG.long. The first four multivariate regression models involved the following covariates: (1) additive model with two covariates CAG.long and CAG.short, (2) a single covariate defined as the sum of CAG lengths (=CAG long + CAG.short), (3) a single covariate defined as the product of CAG lengths (=CAG.long $\times$ CAG.short), and (4) a model with three covariates CAG.long, CAG. short, and their interaction term. Polynomial regression models 5-8 evaluated the quadratic effect of CAG.long. Models 5-7 extended models 1-3, respectively, by adding the square term of CAG.long. Model 8 evaluated three covariates square of CAG.long, CAG.short, and their interaction term. Each model also included age at blood draw and sex. These models were evaluated in three subsets: all individuals, only controls, and only HD mutation carriers. We could not evaluate controls in the Registry-HD lymphoblastoid cells and the MTM fibroblast data due to low sample size $(n<25$ controls)

CCG-repeat length versus HTT methylation. We evaluated five multivariate linear regression models for studying the effect of CCG-repeat length on cg22982173 methylation (dependent variable) in the Registry-HD cohort (up to 372 observations). Model 1 involved two covariates: the two alleles measuring CCG-repeat length. Model 2 extended model 1 by adding two alleles measuring CAG-repeat length. Model 3 is the same as model 1, but limited to the analysis to 209 individuals with homozygosity for $\mathrm{CCG}_{7}$ (where 7 equals the CCG-repeat length). Model 4 involved both alleles for CAG length plus HD disease status. Finally, we re-examined model 5 ( $N=350$ observations) that included both alleles of CAG length, but omitted all HD cases that carried an atypical HTT structure as defined in ref. ${ }^{22}$

Htt Q175 mouse. In the RRBS study, we analyzed HET Htt KI mouse lines Q175 and Q20 mice ${ }^{49}$. The HET $\mathrm{Htt}$ KI line expressed one wild-type endogenous $\mathrm{Htt}$ allele and a second $\mathrm{Htt}$ allele with KI of human $\mathrm{mHTT}$ exon 1 with either $\sim 190$ CAG repeats (Q175) or 20 CAG repeats (Q20). Male HET mice were crossed with C57BL/6J (B6J.zQ175 KI, JAX stock number: 370476, https://www.jax.org/strain/ 370476) female mice at the Jackson Laboratory (Bar Harbor, ME ${ }^{29}$. Animals born within 3-4 days from litters having four to eight pups were identified by ear tags, tail sampled for genotyping, and weaned at $\sim 3$ weeks of age. HET mice were selected based on the CAG repeat sizing to allow a Gaussian distribution of CAG 
repeats in the experimental cohort to avoid skewed distributions. Best Gaussian fit was judged by eye. Body weight cut off: experimental animals had to weigh $>11 \mathrm{~g}$ (females) and $>13 \mathrm{~g}$ (males) by 5 weeks of age. Animals presenting any anomaly were excluded. Unacceptable anomalies were cataracts, malocclusion, missing/ small eye, ear infection, unreadable, or missing tag. Mice were housed in cages enriched with two play tunnels, a plastic bone and enviro-dri ${ }^{\circledR}$ (Shepherd Specialty Papers). Animal cage changes occurred weekly. The cages were maintained on a 12:12 $\mathrm{h}$ light/dark cycle. Water and food were freely available at all times. This study was carried out in strict accordance with the recommendations in the Guide for the Care and Use of Laboratory Animals, NRC (2010). The protocols were approved by the Institutional Animal Care and Use Committee of PsychoGenics, Inc., an AAALAC International accredited institution (Unit \#001213).

The mouse methylation array study was conducted at UCLA. HD KI Q175 mice and wild-type littermate control mice were obtained from The Jackson Laboratory (JAX stock number: 370476). Animals were housed in standard mouse cages under conventional laboratory conditions, with constant temperature and humidity, $12 \mathrm{~h} /$ $12 \mathrm{~h}$ light/dark cycle (7.00 a.m./7.00 p.m.) and food and water ad libitum. All animal studies were carried out in strict accordance with National Institutes of Health guidelines and approved by the UCLA Institutional Animal Care and Use Committees.

Mouse methylation data. For the custom methylation array study (HorvathMammalMethylChip40), we profiled eight tissue samples for each tissue and group defined by disease status (Q175 and Q20). We used the Illumina array for five tissues: cerebellum, striatum, cortex, liver, and blood. In addition, we used another platform RRBS analysis to generated additional methylation data for the cerebellum and striatum (again eight samples per group and tissue). We limited the CpGs with at least 15 observations, yielding 2,896,456 and 2,965,343 CpGs available for the cerebellum and striatum EWAS, respectively. We performed Student's $t$ tests for association tests and combined the results across the tissues based on the Stouffer's method.

RRBS is a widely used technology because it is cost-effective at measuring cytosine methylation data at hundreds of thousands (or even millions) of locations at a single-nucleotide level. Compared to whole-genome sequencing it is cost effective because it combines restriction enzymes and bisulfite sequencing to enrich for areas of the genome with a high $\mathrm{CpG}$ content. Its limitations include a bias towards regions with a high $\mathrm{CpG}$ content and relatively low coverage of individual CpGs (compared to an Illumina array).

The mammalian methylation array is attractive because it provides very high coverage (over thousand $\mathrm{X}$ ) of highly conserved CpGs in mammals, but it focuses on only $37 \mathrm{k} \mathrm{CpGs}$ that are highly conserved across mammals.

An ovine transgenic HD model. We analyzed a total of 168 sheep (57\% females): $84 \mathrm{HD}$ transgenic sheep age matched with 84 sheep as controls. The age of sheep ranged from 2.9 to 7.0 years with mean $\pm \mathrm{SD}=4.1 \pm 0.8$. HD transgenic sheep generated from a new large-animal HD transgenic ovine model ${ }^{24}$. Sheep (Ovis aries L.) were selected because the developmental pattern of the ovine basal ganglia and cortex (the regions primarily affected in HD) is similar to the analogous regions of the human brain. Microinjection of a full-length human HTT cDNA containing 73 polyglutamine repeats under the control of the human promotor resulted in six transgenic founders varying in copy number of the transgene ${ }^{24}$.

To isolate genomic DNA, thawed blood samples $(300 \mu \mathrm{l})$ were treated twice with red cell lysis buffer (300 mM sucrose, $5 \mathrm{mM} \mathrm{MgCl} 2,10 \mathrm{mM}$ Tris $\mathrm{pH} 8,1 \%$ Triton X-100), for $10 \mathrm{~min}$ on ice; each incubation was followed by centrifugation at 1800 r.c.f. for $10 \mathrm{~min}$ at $4^{\circ} \mathrm{C}$, and removal of supernatant. Final pellet was incubated in cell digestion buffer ( $2.4 \mathrm{mM}$ EDTA, $75 \mathrm{mM} \mathrm{NaCl}, 0.5 \%$ sodium dodecyl sulfate) and proteinase $\mathrm{K}$ (final concentration $500 \mu \mathrm{g} / \mathrm{ml}$ ) for at least $2 \mathrm{~h}$ at $50^{\circ} \mathrm{C}$. An equal volume of phenol:chloroform:isoamyl alcohol $(25: 24: 1 ; \mathrm{pH} 8)$ was added and samples mixed by inversion, followed by centrifugation at 14,000 r.pm. for $5 \mathrm{~min}$ at room temperature. This step was repeated if required. Supernatant was collected and mixed with $2 \times$ volume $100 \%$ ethanol to precipitate DNA. Following removal and evaporation of residual ethanol, genomic DNA samples were resuspended in $50 \mu \mathrm{l}$ TE buffer ( $\mathrm{pH}$ 8). Sample concentrations were initially measured on nanodrop to inform further dilution of samples to range of 100-1000 $\mu \mathrm{g}$ for measurement on Qubit. All protocols used were approved by the University of Auckland Animal Ethics Committee (New Zealand) and the SARDI/PIRSA (South Australian Research and Development Institute/Primary Industries and Regions South Australia) Animal Ethics Committee (Approval number 19/02). Moreover, all work involving OVT73 Sheep was approved by PIRSA Animal Ethics Committee with oversite from the University of Auckland Animal Ethics Committee. DNAm arrays were profiled using a custom Illumina methylation array (HorvathMammalMethyl40) based on 38,000 CpG sites in highly conserved regions in mammals. EWAS was performed using the R function "standardScreeningBinaryTrait" from the "WGCNA" R package ${ }^{50}$

HD motor progression in the Enroll-HD data 1. To establish a measure of HD motor progression, we used the large-scale Enroll-HD database comprising 14,850 longitudinal observations across 5204 manifest HD individuals. Of the 5204 patients, 312 were initially in pre-manifest then converted to manifest disease status, in which we only used the visits since manifest phase. We applied linear mixed models under R/nlme 3.1-148 with random effects to longitudinal measures of UHDRS total motor scores, adjusted for age, sex, CAG-repeat length, age at motor onset, education attainment, and visit time as fixed effects. The UHDRS total motor score is a sum of 31 items across oculomotor function, dysarthria, chorea, dystonia, gait, and postural stability. Each item is a scale from 0 to 4 indicating no abnormalities (score as 0 ) to the most severe impairment (score as 4). The possible range of the total motor score is $0-124$. We restricted our analysis for the observations with motor scores $\geq 5$. The random effects included a random intercept with respect to individuals and a random slope with respect to visit to account for heterogeneous changes in motor scores across individuals that could not be captured by the fixed effect of visit. Therefore, we computed the empirical Bayes estimates of the random slopes as a "raw" measure of HD motor progression. Next, we regressed the random slopes on sex, age at baseline, CAG-repeat length, age at motor onset and education attainment, and used the residuals as the measure of HD progression for our downstream analysis. The mean (SD) of follow-up estimates were 2.1 (1.03) years in all 5204 manifest individuals and 3.13 (1.27) years in the subset of 354 individuals available with DNAm array data.

HD progression in Enroll-HD data 2. In an analogous manner, we performed the linear mixed analysis to establish the measure of $\mathrm{HD}$ motor progression, using the manifest individuals in the Enroll-HD data 2. The data 2 aimed to profile two longitudinal measures of DNAm array with approximately a 7-year gap; thus, most of the first blood samples were collected during visits by the individuals in the Registry-HD study. We removed the individuals already appearing in the Enroll$\mathrm{HD}$ data and the visits with motor scores $<5$, leaving 275 manifest individuals (including 73 converting to manifest) remaining in our progression analysis. In establishing the measure of $\mathrm{HD}$ progression, there were $>1860$ observations across the 275 individuals available for our analysis, with a mean follow-up period of $\sim 7.9$ years, from the visits aligned with the first DNAm methylation profiles through to the most recent visits. The model framework of the linear mixed analysis was similar to the one used for Enroll-HD data 1, whereas the age at fixed effect was based on the visit aligned with the first methylation profiles. The random slopes with respect to visits were also adjusted for sex, age, CAG-repeat length, age at motor onset, and education attainment for the downstream analysis.

Registry-HD data. To establish the HD motor progression scores, we used the cross-sectional phase motor scores based on the last visits, similar to what other authors had done for the Registry-HD data ${ }^{13}$. We applied linear regression analysis to the motor scores, adjusted for sex, age at the last visit, age at motor onset, age at DNAm profiles, CAG length and education attainment, and used the residuals as the measure of the progression for downstream analysis.

Assessment of HD progression in HTT cg22982173. We studied the implication of HTT cg22982173 in HD progression across different clinical assessment domains. While our primary measure of HD progression was based on motor score assessments across several visits following the blood draw, we also defined analogous measures of progression based on other clinical assessments: functional assessment score, and the Stroop color and word tests.

Detailed visualization of regional EWAS coMET. We applied the R package comet (version 3.1$)^{51}$ to visualize the genomic regions of interest from our EWAS results.

EWAS of HD motor progression. We conducted the EWAS of motor progression scores based on the adjusted random slopes on Enroll-HD data 1 and data 2, respectively, and based on the adjusted last motor scores on the Registry-HD data. EWAS was performed with the function "standardScreeningNumericTrait" in the WGCNA R package under R 3.4.3. Effect sizes were based on biweight midcorrelation (bicor) for robust correlation estimates ${ }^{50}$. We combined the results across the three studies via fixed-effect models weighted by inverse variance under METAL (released 2011-03-25) ${ }^{52}$ and only used the overlap CpGs between the Illumina $450 \mathrm{k}$ and EPIC array.

Gaphunter analysis to detect confounding by SNPs. The Gaphunter hunter software allows one to identify CpG probes that are confounded by adjacent $\mathrm{SNPs}^{23}$. We applied minfi/Gaphunter (3.6) under R to our blood DNAm data from Enroll-HD data 1, Enroll-HD data 2, and Registry-HD, respectively. The analysis was performed using the $\mathrm{R}$ minfi/gapfunction function, which identifies $\mathrm{CpG}$ probes with a gap in a (methylation) beta signal greater than or equal to the defined threshold and reports number of clusters constituting the gap. We used the default threshold of 0.05 and a default cut off value of 0.01. Evidence for SNP confounding exists if the $\mathrm{CpG}$ exhibits at least two distinct clusters that involve $>20$ individuals each. Gap patterns were examined in the $33 \mathrm{CpGs}$ associated with $\mathrm{HD}$ disease status and the three CpGs associated with motor progression manifest HD cases.

Functional enrichment analysis. The anRichment software is implemented in the online tool HDinHD ${ }^{29}$ (www.HDinHD.org), which includes published gene sets, 
including GO (gene ontology) terms $s^{53}$, KEGG (Kyoto Encyclopedia of Genes and Genomes) pathways ${ }^{54}$, Molecular Signatures Database gene sets ${ }^{55}$, and curated literature gene sets included in the "userListEnrichment" function ${ }^{56}$ of the WGCNA (3.6.3) R package as well as transcriptomic co-expression network modules identified in analyses of publicly available expression data from HD patients as well as mouse models.

The GOMETH enrichment analysis properly takes into account the different number of probes per gene present on Illumina array (450k in our analysis) by using Wallenius' noncentral hypergeometric test ${ }^{30}$. We used the GOMETH R function ( $\mathrm{R}$ version 3.6), which includes GO terms and KEGG pathways.

Reporting summary. Further information on research design is available in the Nature Research Reporting Summary linked to this article.

\section{Data availability}

Our data are available from two data repositories. First, Gene Expression Omnibus (Superseries GSE147004 and subseries GSE146917, GSE147002, GSE147003, and GSE72778 (human brain methylation). Second, from Enroll-HD [https://www.enroll-hd. org/]. Please direct inquiries to info@chdifoundation.org with the words "Enroll-HD Methylation data" in the subject line. All other relevant data supporting the key findings of this study are available within the article and its Supplementary information files or from the corresponding author upon reasonable request. A reporting summary for this Article is available as a Supplementary information file.

\section{Code availability}

This article does not use any custom code or mathematical algorithm. Relevant code or software has been published in previous articles ("Methods"). In addition, software code is available upon request.

Received: 24 October 2019; Accepted: 14 August 2020;

Published online: 10 September 2020

\section{References}

1. Orr, H. T. \& Zoghbi, H. Y. Trinucleotide repeat disorders. Annu. Rev. Neurosci. 30, 575-621 (2007).

2. Djousse, L. et al. Interaction of normal and expanded CAG repeat sizes influences age at onset of Huntington disease. Am. J. Med. Genet. A 119A, 279-282 (2003).

3. Ross, C. A. et al. Huntington disease: natural history, biomarkers and prospects for therapeutics. Nat. Rev. Neurol. 10, 204-216 (2014).

4. Gusella, J. F. \& Macdonald, M. Genetic criteria for Huntington's disease pathogenesis. Brain Res. Bull. 72, 78-82 (2007).

5. Lee, J.-M. et al. CAG repeat not polyglutamine length determines timing of Huntington's disease onset. Cell 178, 887-900.e14 (2019).

6. Horvath, S. et al. Huntington's disease accelerates epigenetic aging of human brain and disrupts DNA methylation levels. Aging 8, 1485-1512 (2016).

7. Villar-Menendez, I. et al. Increased 5-methylcytosine and decreased 5hydroxymethylcytosine levels are associated with reduced striatal A2AR levels in Huntington's disease. Neuromol. Med. 15, 295-309 (2013).

8. De Souza, R. A. et al. DNA methylation profiling in human Huntington's disease brain. Hum. Mol. Genet. 25, 2013-2030 (2016).

9. Ng, C. W. et al. Extensive changes in DNA methylation are associated with expression of mutant huntingtin. Proc. Natl. Acad. Sci. USA 110, 2354-2359 (2013).

10. Landwehrmeyer, G. B. et al. Data Analytics from Enroll-HD, a global clinical research platform for Huntington's disease. Mov. Disord. Clin. Pract. 4 , 212-224 (2017).

11. Orth, M. et al. Observing Huntington's disease: the European Huntington's Disease Network's REGISTRY. PLoS Curr. 2, RRN1184 (2010).

12. Huntington Study Group. Unified Huntington's Disease Rating Scale: reliability and consistency. Mov. Disord. 11, 136-142 (1996).

13. Moss, D. J. H. et al. Identification of genetic variants associated with Huntington's disease progression: a genome-wide association study. Lancet Neurol. 16, 701-711 (2017)

14. Horvath, S. DNA methylation age of human tissues and cell types. Genome Biol. 14, R115 (2013).

15. Hannum, G. et al. Genome-wide methylation profiles reveal quantitative views of human aging rates. Mol. Cell 49, 359-367 (2013).

16. Horvath, S. et al. Epigenetic clock for skin and blood cells applied to Hutchinson Gilford Progeria Syndrome and ex vivo studies. Aging 10, 1758-1775 (2018).
17. Levine, M. E., Lu, A. T., Bennett, D. A. \& Horvath, S. Epigenetic age of the pre-frontal cortex is associated with neuritic plaques, amyloid load, and Alzheimer's disease related cognitive functioning. Aging 7, 1198-1211 (2015).

18. Lu, A. T. et al. DNA methylation GrimAge strongly predicts lifespan and healthspan. Aging 11, 303-327 (2019).

19. Horvath, S. et al. An epigenetic clock analysis of race/ethnicity, sex, and coronary heart disease. Genome Biol. 17, 171 (2016)

20. Mansell, G. et al. Guidance for DNA methylation studies: statistical insights from the Illumina EPIC array. BMC Genomics 20, 366 (2019).

21. Panegyres, P. K., Beilby, J., Bulsara, M., Toufexis, K. \& Wong, C. A study of potential interactive genetic factors in Huntington's disease. Eur. Neurol. 55, 189-192 (2006).

22. Ciosi, M. et al. A genetic association study of glutamine-encoding DNA sequence structures, somatic CAG expansion, and DNA repair gene variants, with Huntington disease clinical outcomes. EBioMedicine 48, 568-580 (2019).

23. Andrews, S. V., Ladd-Acosta, C., Feinberg, A. P., Hansen, K. D. \& Fallin, M. D. "Gap hunting" to characterize clustered probe signals in Illumina methylation array data. Epigenet. Chromatin 9, 56 (2016).

24. Jacobsen, J. C. et al. An ovine transgenic Huntington's disease model. Hum. Mol. Genet. 19, 1873-1882 (2010).

25. Chiang, C. et al. Complex reorganization and predominant non-homologous repair following chromosomal breakage in karyotypically balanced germline rearrangements and transgenic integration. Nat. Genet. 44(Suppl. 1), 390-397 (2012).

26. Handley, R. R. et al. Metabolic disruption identified in the Huntington's disease transgenic sheep model. Sci. Rep. 6, 20681 (2016).

27. Huntington's Disease Sheep Collaborative Research, G. et al. Further molecular characterisation of the OVT73 transgenic sheep model of Huntington's disease identifies cortical aggregates. J. Huntington's Dis. 2, 279-295 (2013).

28. Skene, D. J. et al. Metabolic profiling of presymptomatic Huntington's disease sheep reveals novel biomarkers. Sci. Rep. 7, 43030 (2017).

29. Langfelder, P. et al. Integrated genomics and proteomics define huntingtin CAG length-dependent networks in mice. Nat. Neurosci. 19, 623-633 (2016).

30. Phipson, B., Maksimovic, J. \& Oshlack, A. missMethyl: an R package for analyzing data from Illumina's HumanMethylation 450 platform. Bioinformatics 32, 286-288 (2015).

31. Libertini, E. et al. Saturation analysis for whole-genome bisulfite sequencing data. Nat. Biotechnol. 34, 691-693 (2016).

32. van Eijk, K. et al. Genetic analysis of DNA methylation and gene expression levels in whole blood of healthy human subjects. BMC Genomics 13, 636 (2012).

33. Garrick, D., Fiering, S., Martin, D. I. K. \& Whitelaw, E. Repeat-induced gene silencing in mammals. Nat. Genet. 18, 56-59 (1998).

34. Ng, C. W. et al. Extensive changes in DNA methylation are associated with expression of mutant huntingtin. Proc. Natl. Acad. Sci. USA 110, 2354-2359 (2013).

35. Wilson, C., Bellen, H. J. \& Gehring, W. J. Position effects on eukaryotic gene expression. Annu. Rev. Cell Biol. 6, 679-714 (1990).

36. Rexroad, C. E. Transgenic technology in animal agriculture. Anim. Biotechnol. 3, 1-13 (1992).

37. Santoro, M. R., Bray, S. M. \& Warren, S. T. Molecular mechanisms of Fragile X syndrome: a twenty-year perspective. Annu. Rev. Pathol. 7, 219-245 (2012).

38. He, F. \& Todd, P. K. Epigenetics in nucleotide repeat expansion disorders. Semin. Neurol. 31, 470-483 (2011).

39. Castaldo, I. et al. DNA methylation in intron 1 of the frataxin gene is related to GAA repeat length and age of onset in Friedreich ataxia patients. J. Med. Genet. 45, 808-812 (2008).

40. Sun, J. H. et al. Disease-associated short tandem repeats co-localize with chromatin domain boundaries. Cell 175, 224-238.e15 (2018).

41. Pinto, R. M. et al. Mismatch repair genes Mlh1 and Mlh3 modify CAG instability in Huntington's disease mice: genome-wide and candidate approaches. PLoS Genet. 9, e1003930 (2013).

42. Gillis, J. et al. The DNAJB6 and DNAJB8 protein chaperones prevent intracellular aggregation of polyglutamine peptides. J. Biol. Chem. $\mathbf{2 8 8}$ 17225-17237 (2013).

43. Kakkar, V. et al. The S/T-rich motif in the DNAJB6 chaperone delays polyglutamine aggregation and the onset of disease in a mouse model. Mol. Cell 62, 272-283 (2016)

44. Mielcarek, M. et al. HDAC4 reduction: a novel therapeutic strategy to target cytoplasmic Huntingtin and ameliorate neurodegeneration. PLoS Biol. 11, e1001717 (2013).

45. Lowry, E. R., Kruyer, A., Norris, E. H., Cederroth, C. R. \& Strickland, S. The GluK4 kainate receptor subunit regulates memory, mood, and excitotoxic neurodegeneration. Neuroscience 235, 215-225 (2013). 
46. Triche, T. J., Weisenberger, D. J., Van Den Berg, D., Laird, P. W. \& Siegmund, K. D. Low-level processing of Illumina Infinium DNA Methylation BeadArrays. Nucleic Acids Res. 41, e90-e90 (2013).

47. Chen, B. H. et al. DNA methylation-based measures of biological age: metaanalysis predicting time to death. Aging 8, 1844-1865 (2016).

48. Aulchenko, Y. S., Ripke, S., Isaacs, A. \& van Duijn, C. M. GenABEL: an R library for genome-wide association analysis. Bioinformatics 23, 1294-1296 (2007).

49. Menalled, L. B. et al. Comprehensive behavioral and molecular characterization of a new knock-in mouse model of Huntington's disease: zQ175. PLoS ONE 7, e49838 (2012).

50. Langfelder, P. \& Horvath, S. WGCNA: an R package for weighted correlation network analysis. BMC Bioinform. 9, 559 (2008).

51. Martin, T. C., Yet, I., Tsai, P. C. \& Bell, J. T. coMET: visualisation of regional epigenome-wide association scan results and DNA co-methylation patterns. BMC Bioinform. 16, 131 (2015).

52. Willer, C. J., Li, Y. \& Abecasis, G. R. METAL: fast and efficient meta-analysis of genomewide association scans. Bioinformatics 26, 2190-2191 (2010).

53. Blake, J. A. et al. Gene Ontology Consortium: going forward. Nucleic Acids Res. 43, D1049-D1056 (2015).

54. Kanehisa, M., Sato, Y., Kawashima, M., Furumichi, M. \& Tanabe, M. KEGG as a reference resource for gene and protein annotation. Nucleic Acids Res. 44, D457-D462 (2016).

55. Subramanian, A. et al. Gene set enrichment analysis: a knowledge-based approach for interpreting genome-wide expression profiles. Proc. Natl. Acad. Sci. USA 102, 15545-15550 (2005).

56. Miller, J. A. et al. Strategies for aggregating gene expression data: the collapseRows R function. BMC Bioinform. 12, 322 (2011).

57. Aryee, M. J. et al. Minfi: a flexible and comprehensive Bioconductor package for the analysis of Infinium DNA methylation microarrays. Bioinformatics $\mathbf{3 0}$, 1363-1369 (2014).

\section{Acknowledgements}

Enroll-HD is a longitudinal observational study for Huntington's disease families intended to accelerate progress towards therapeutics. This research was supported by CHDI Foundation, Inc., a non-profit biomedical research organization exclusively dedicated to developing therapeutics for HD. Enroll-HD would not be possible without the vital contribution of the research participants and their families. The European Huntington's Disease Network (EHDN) provided DNA samples underlying the RegistryHD dataset. Individuals who contributed to the collection of the Enroll-HD data are listed in Supplementary Data 5. Data used in this work included MTM Study participantspecific data as well as data derived from biosamples generously provided by those participants, and were made available by Dr. Sarah Tabrizi (University College London), Principal Investigator, and Dr. Michael Orth (University Hospital of Ulm), Principal Investigator. The transgenic sheep pedigree information and blood sample collection was facilitated by Dr Suzanne Reid (The University of Auckland).

\section{Author contributions}

S.H., X.W.Y., R.G.S., D.M., and A.T.L. conceived of the study. A.T.L. carried out most of the statistical analyses. P.N., M.J.G., C.S.B., S.R.R., T.A.H., and R.G.S. conceived and carried out the experiments of ovine transgenic Huntington's disease model. N.W. and X.W.Y. conceived and carried out the experiments of Htt Q175 mouse model. P.L. carried out gene enrichment analysis and system biology-related analysis. G.C. helped brain-based methylation data analysis, M.C., A.C., A.M., and D.M. designed and carried out the implications of glutamine-encoding DNA sequence structures in HD methylation patterns. S.K., H.W., R.C., J.C., J.A., J.R., A.P., and T.F.V. contributed data, helped with the write up, and participated in the interpretation of the results. All authors participated in the interpretation of the study and helped revise the article.

\section{Competing interests}

UC Regents (the employer of S.H. and A.T.L.) has filed patents surrounding several epigenetic biomarkers of aging (including GrimAge), which list S.H. and A.T.L. as inventors.

\section{Additional information}

Supplementary information is available for this paper at https://doi.org/10.1038/s41467020-18255-5.

Correspondence and requests for materials should be addressed to S.H.

Peer review information Nature Communications thanks the anonymous reviewer(s) for their contribution to the peer review of this work.

Reprints and permission information is available at http://www.nature.com/reprints

Publisher's note Springer Nature remains neutral with regard to jurisdictional claims in published maps and institutional affiliations.

(c) (i) Open Access This article is licensed under a Creative Commons Attribution 4.0 International License, which permits use, sharing, adaptation, distribution and reproduction in any medium or format, as long as you give appropriate credit to the original author(s) and the source, provide a link to the Creative Commons license, and indicate if changes were made. The images or other third party material in this article are included in the article's Creative Commons license, unless indicated otherwise in a credit line to the material. If material is not included in the article's Creative Commons license and your intended use is not permitted by statutory regulation or exceeds the permitted use, you will need to obtain permission directly from the copyright holder. To view a copy of this license, visit http://creativecommons.org/ licenses/by/4.0/.

(C) The Author(s) 2020 